\title{
Euler Solutions of Pseudodifferential Equations
}

\author{
Bert-Wolfgang Schulze \\ Nikolai N. Tarkhanov* \\ Institut für Mathematik \\ Universität Potsdam \\ Postfach 601553 \\ 14415 Potsdam \\ Germany
}

March 12, 1998

${ }^{*}$ Supported by the Max-Planck Gesellschaft. 


\begin{abstract}
We consider a homogeneous pseudodifferential equation on a cylinder $\mathcal{C}=\mathbb{R} \times X$ over a smooth compact closed manifold $X$ whose symbol extends to a meromorphic function on the complex plane with values in the algebra of pseudodifferential operators over $X$. When assuming the symbol to be independent on the variable $t \in \mathbb{R}$, we show an explicit formula for solutions of the equation. Namely, to each non-bijectivity point of the symbol in the complex plane there corresponds a finite-dimensional space of solutions, every solution being the residue of a meromorphic form manufactured from the inverse symbol. In particular, for differential equations we recover Euler's theorem on the exponential solutions. Our setting is model for the analysis on manifolds with conical points since $\mathcal{C}$ can be thought of as a 'stretched' manifold with conical points at $t=-\infty$ and $t=\infty$.
\end{abstract}

AMS subject classification: primary: 45N05; secondary: $34 \mathrm{G} 10$.

Key words and phrases: pseudodifferential operator, meromorphic family, residue. 


\section{Contents}

Introduction $\quad 4$

1 Meromorphic families $\quad 6$

$\begin{array}{lll}2 & \text { Characteristic values } & 7\end{array}$

$\begin{array}{lll}3 & \text { Factorisation } & 10\end{array}$

4 Resolvent $\quad 13$

5 Unitary reduction $\quad 15$

$\begin{array}{lll}6 & \text { Inhomogeneous equation } & 17\end{array}$

$\begin{array}{lll}7 & \text { Transposed equation } & 24\end{array}$

$\begin{array}{llr}8 & \text { Index } & 28\end{array}$

$\begin{array}{ll}\text { References } & 31\end{array}$ 


\section{Introduction}

The aim of this paper is to bring together two areas in which the Euler theory for ordinary differential equations with constant coefficients is a powerful source of intuition and an important ingredient. One of the two deals with abstract meromorphic functions taking their values in the space of bounded operators between Banach spaces. This area was intensively studied in the late '60s by Blekher [Ble69], Krein and Trofimov [KT69], Eni [Eni69], Sigal [Sig70], Markus and Sigal [MS70], Gokhberg and Sigal [GS71] etc., who developed the earlier papers of Keldysh [Kel51] and Gokhberg [Gok51]. The other area is the analysis of pseudodifferential operators on manifolds with conical points. It is originated with the paper of Kondrat'ev [Kon67] and was developed by Plamenevskii [Pla89], Schulze [Sch91, Sch98], Melrose and Mendoza [MM83], Schrohe and Schulze [SS94, SS95], Maz'ya, Kozlov and Rossmann [MKR97] and other authors. The definition of a pseudodifferential operator close to a conical point relies on the concept of a parameterdependent pseudodifferential operator on a smooth closed manifold, as is introduced by Agranovich and Vishik [AV64]. On the other hand, the key result of the theory is an asymptotic expansion of solutions near conical points, the idea going back at least as far as Evgrafov [Evg61], Agmon and Nirenberg [AN63], Kondrat'ev [Kon67] and Maz'ya and Plamenevskii [MP72]. If pulled back to $t=\infty$ by the diffeomorphism $t \mapsto e^{-t}$, the asymptotics are nothing but Euler solutions to the equation defined by the conormal symbol at the conical point. This latter is a parameter-dependent pseudodifferential operator on a cross-section of the manifold in a neighbourhood of the conical point, the parameter being the covariable $\tau \in \mathbb{R}$ of $t$. Moreover, it extends meromorphically in $\tau$ to a neighbourhood of the real axis. In the present work we will thus be concerned with an equation

$$
\frac{1}{2 \pi} \int_{\Gamma_{\gamma}} d z \int_{\mathbb{R}} e^{i\left(t-t^{\prime}\right) z} a(z) u\left(t^{\prime}\right) d t^{\prime}=f(t), \quad t \in \mathbb{R}
$$

on the cylinder $\mathcal{C}=\mathbb{R} \times X$ over a $C^{\infty}$ compact closed manifold $X$. Here, $a(z)$ is a meromorphic function in a strip $\Xi=\{z \in \mathbb{C}: \Im z \in(a, b)\}$ containing the line $\Gamma_{\gamma}=\mathbb{R}+i \gamma$, which takes its values in the space of classical pseudodifferential operators of order $m$ on $X$. We assume that $\Gamma_{\gamma}$ contains no pole of $a(z)$ and that the restriction of $a(z)$ to each horizontal line within the strip $\Xi$ behaves like a parameter-dependent pseudodifferential operator on $X$. When first defined on functions $u \in C_{\text {comp }}^{\infty}\left(\mathbb{R}, C^{\infty}(X)\right)$, the operator on the left-hand side of $(0.1)$ extends to a mapping of weighted Sobolev spaces on the cylinder, $H^{s, \gamma}(\mathcal{C}) \rightarrow H^{s-m, \gamma}(\mathcal{C})$, for every $s \in \mathbb{R}$. Thus, we may take $H^{s-m, \gamma}(\mathcal{C})$ as a domain for $f$ and $H^{s, \gamma}(\mathcal{C})$ as such for $u$. Under the natural condition of ellipticity, we give an explicit formula for the resolvent $a^{-1}(z)$ 
and prove that equation (0.1) has a unique solution $u$ for each right-hand side $f$. We then use this result to investigate equation (0.1) on weighted Sobolev spaces $H^{s, w}(\mathcal{C})$, with $w=\left(w_{-}, w_{+}\right)$a pair of real numbers controlling the growth of functions at $t= \pm \infty$. This scale of Sobolev spaces on the cylinder is more capacious to specify the solutions of $(0.1)$ than the oneparameter scale. In particular, taking $w=(-\gamma, \gamma)$ yields $H^{s, w}(\mathcal{C})=H^{s, \gamma}(\mathcal{C})$ for all $s, \gamma \in \mathbb{R}$. Generally speaking, the operator on the left-hand side of (0.1) can not be extended to a continuous mapping $H^{s, w}(\mathcal{C}) \rightarrow H^{s-m, w}(\mathcal{C})$, even if $w_{ \pm} \in(a, b)$. To give meaning to $(0.1)$ we distinguish between the cases $-w_{-}<w_{+}$and $-w_{-}>w_{+}$. If $-w_{-}<w_{+}$, then in order that a function $u$ belong to $H^{s, w}(\mathcal{C})$ it is necessary and sufficient that $u \in H^{s, \gamma}(\mathcal{C})$ for each $-w_{-}<\gamma<w_{+}$. Moreover, for any $u \in H^{s, w}(\mathcal{C})$, the Fourier transform $\mathcal{F} u(z)=\int_{\mathbb{R}} e^{-i z t^{\prime}} u\left(t^{\prime}\right) d t^{\prime}$ is a holomorphic function in the strip $-w_{-}<\Im z<w_{+}$with values in $H^{s}(X)$. Denote by Dom $A$ the subspace of $H^{s, w}(\mathcal{C})$ consisting of all $u$ with the property that $\operatorname{res}_{p} e^{i t z} a(z) \mathcal{F} u(z)=0$ at each pole $p$ of $a(z)$ in the strip $-w_{-}<\Im z<w_{+}$. It is fairly straightforward that $\operatorname{Dom} A$ is of finite codimension. For any $u \in \operatorname{Dom} A$, the integral on the left-hand side of $(0.1)$ is independent of the particular choice of $\gamma$ in the interval $\left(-w_{-}, w_{+}\right)$. Moreover, it gives a function in $H^{s-m, w}(\mathcal{C})$ thus defining an operator $A: \operatorname{Dom} A \rightarrow H^{s-m, w}(\mathcal{C})$. We prove that if $a(z)$ is invertible on both $\Gamma_{-w_{-}}$and $\Gamma_{w_{+}}$, then the equation $A u=f$ has a unique solution $u \in \operatorname{Dom} A$ for every $f$ in a subspace of $H^{s-m, w}(\mathcal{C})$ of finite codimension. In particular, $A$ is a Fredholm operator. Let us now turn to the case $-w_{-}>w_{+}$. Were $A$ a continuous linear operator $H^{s, w}(\mathcal{C}) \rightarrow H^{s-m, w}(\mathcal{C})$, the transpose $A^{\prime}$ would define an operator $H^{-s+m,-w}(\mathcal{C}) \rightarrow H^{-s,-w}(\mathcal{C})$, where $-w=\left(-w_{-},-w_{+}\right)$. Note that the couple $-w$ already meets the condition $w_{-}<-w_{+}$, and so we may apply the above arguments again, with $a(z)$ replaced by $a^{\prime}(-z)$, to arrive at an operator $A^{\prime}: \operatorname{Dom} A^{\prime} \rightarrow H^{-s,-w}(\mathcal{C})$. The domain of $A^{\prime}$ is a subspace of $H^{-s+m,-w}(\mathcal{C})$ of finite codimension which is non-zero unless $a(z)$ has no pole in the strip $w_{+}<\Im z<-w_{-}$. Hence it follows, for $u \in H^{s, w}(\mathcal{C})$, that $\left(A^{\prime}\right)^{\prime} u$ is determined uniquely up to elements of the annihilator of Dom $A^{\prime}$ in $H^{s-m, w}(\mathcal{C})$. As this annihilator is finitedimensional, the transpose of $A^{\prime}$ is defined modulo operators of finite rank. We set $A=\left(A^{\prime}\right)^{\prime}$ for any one choice of the operators on $H^{s, w}(\mathcal{C})$ taking their values in the annihilator of $\operatorname{Dom} A^{\prime}$ in $H^{s-m, w}(\mathcal{C})$. Then $A$ is welldefined as mapping $H^{s, w}(\mathcal{C}) \rightarrow H^{s-m, w}(\mathcal{C})$ and the definition agrees with the usual one in the case of differential operators. We prove that if $a(z)$ is invertible on both $\Gamma_{-w_{-}}$and $\Gamma_{w_{+}}$, then the equation $A u=f$ has a solution $u \in H^{s, w}(\mathcal{C})$ for each $f \in H^{s-m, w}(\mathcal{C})$. Moreover, the space of solutions of the corresponding homogeneous equation is finite-dimensional, i.e., $A$ is a Fredholm operator. In both the cases we show an index formula for $A$ which turns out to be a version of the logarithmic residue theorem of Gokhberg and Sigal [GS71]. Let us finally remark that our results extend easily to the case where $X$ is a $C^{\infty}$ compact manifold with boundary. On such a manifold live parameter-dependent boundary value problems with the transmission 
property. Hence we may consider meromorphic functions $a(z)$ in the strip $\Xi$ taking their values in the boundary value problems. The inverse (resolvent) of an elliptic meromorphic function is available in the same class, and so our arguments still go in this context. The necessary tools are developed in Schrohe and Schulze [SS94, SS95].

\section{Meromorphic families}

Let $\Psi_{c l}^{m}(X)$ stand for the space of classical pseudodifferential operators of order $m$ on $X$.

By a parameter-dependent classical pseudodifferential operator of order $m$ on $X$, with parameter $\tau \in \mathbb{R}$, is meant any family $a(\tau)$ of operators in $\Psi_{c l}^{m}(X)$ with the property that $\tau$ enters into the symbol of $a(\tau)$ as an additional covariable. The space of such operators is denoted by $\Psi_{c l}^{m}(X ; \mathbb{R})$.

The space $\Psi_{\mathrm{cl}}^{m}(X)$ bears a natural Fréchet topology. Hence, we may consider holomorphic functions in the strip $\Xi$ taking their values in $\Psi_{\mathrm{cl}}^{m}(X)$. Denote by $\mathcal{A}^{m}(\Xi)$ the space of all holomorphic functions $h(z)$ in $\Xi$ with values in $\Psi_{\mathrm{cl}}^{m}(X)$, such that $h(\tau+i \gamma) \in \Psi_{\mathrm{cl}}^{m}(X ; \mathbb{R})$ uniformly in $\gamma$ on compact segments in $(a, b)$.

Proposition 1.1 For each $a(\tau) \in \Psi_{\mathrm{cl}}^{m}(X ; \mathbb{R})$ there exists a function $h(z) \in \mathcal{A}^{m}(\mathbb{C})$ such that $h(\tau)=a(\tau) \operatorname{modulo} \Psi^{-\infty}(X ; \mathbb{R})$.

Proof. Cf. Theorem 2.2.8 in Schulze [Sch98].

Note that if $h \in \mathcal{A}^{m}(\Xi)$ and $h(\tau+i \gamma) \in \Psi^{-\infty}(X ; \mathbb{R})$ for some $\gamma \in(a, b)$, then $h \in \mathcal{A}^{-\infty}(\Xi)$.

Recall that $a(\tau) \in \Psi_{\mathrm{cl}}^{m}(X ; \mathbb{R})$ is said to be parameter-dependent elliptic if $\sigma^{m}(a)(x ; \tau, \xi) \neq 0$ for each $x \in X$ and all $(\tau, \xi) \in \mathbb{R} \times T_{x}^{*}(X)$ different from zero. If $h \in \mathcal{A}^{m}(\Xi)$ and $h(\tau+i \gamma)$ is parameter-dependent elliptic for some $\gamma \in(a, b)$, then so is the restriction of $h(z)$ to each horizontal line within the strip $\Xi$. Indeed, the principal symbol $\sigma^{m}(h(\tau+i \gamma))$ is independent of $\gamma \in(a, b)$.

We will also consider meromorphic functions in the strip $\Xi$ taking their values in $\Psi_{c l}^{m}(X)$. We restrict our attention to those having a finite number of poles in each strip $\alpha \leq \Im z \leq \beta$ with $a<\alpha \leq \beta<b$. Let $\mathcal{M}^{m}(\Xi)$ stand for the space of all such functions $a(z)$ fulfilling moreover the following properties:

- for each excision function $\chi(z)$ for the set of poles of $h(z)$, we have $(\chi a)(\tau+i \gamma) \in \Psi_{\mathrm{cl}}^{m}(X ; \mathbb{R})$ uniformly in $\gamma$ on compact segments in $(a, b)$;

- close to a pole $p \in \Xi$, we have $a(z)=\sum_{j=-\mu}^{-1} a_{j}(z-p)^{j}+h(z)$ with $a_{j}$ operators of finite rank in $\Psi^{-\infty}(X)$ and $h(z)$ a holomorphic function near $p$ with values in $\Psi_{c l}^{m}(X)$. 
Proposition 1.2 When topologising $\mathcal{A}^{m}(\Xi)$ and $\mathcal{M}^{m}(\Xi)$ in a natural way, we have $\mathcal{M}^{m}(\Xi)=\mathcal{M}^{-\infty}(\Xi)+\mathcal{A}^{m}(\Xi)$ in the sense of non-direct sum of Fréchet spaces.

Proof. Cf. Theorem 5 in Schulze [Sch91, 2.1.2] or Theorem 4.1.8 in Schrohe and Schulze [SS94].

The spaces $\mathcal{M}^{m}(\Xi)$ inherit an "algebra" structure under the pointwise composition of pseudodifferential operators on $X$.

Proposition 1.3 If $a(z) \in \mathcal{M}^{m}(\Xi)$ and $b(z) \in \mathcal{M}^{n}(\Xi)$, then $a(z) b(z) \in$ $\mathcal{M}^{m+n}(\Xi)$.

Proof. Cf. Proposition 6 in Schulze [Sch91, 2.1.2] or Proposition 4.1.7 in Schrohe and Schulze [SS94].

Let $a(z) \in \mathcal{M}^{m}(\Xi)$. Write $a(z)=a_{s}(z)+a_{r}(z)$ by Proposition 1.2, where $a_{s}(z) \in \mathcal{M}^{-\infty}(\Xi)$ and $a_{r}(z) \in \mathcal{A}^{m}(\Xi)$. We say that $a(z)$ is parameterdependent elliptic if so is $a_{r}(z)$. From what has already been said it follows that this definition is correct, i.e., independent of the particular choice of the split of $a$.

Proposition 1.4 Suppose $a(z) \in \mathcal{M}^{m}(\Xi)$ is parameter-dependent elliptic. Then $a(z)$ is invertible away from a discrete subset of $\Xi$ which meets every strip $\alpha \leq \Im z \leq \beta$, with $a<\alpha \leq \beta<b$, only at a finite number of points. Moreover, $a^{-1}(z) \in \mathcal{M}^{-m}(\Xi)$.

Proof. Write $a(z)=a_{s}(z)+h(z)$ with some $a_{s}(z) \in \mathcal{M}^{-\infty}(\Xi)$ and $h(z) \in \mathcal{A}^{m}(\Xi)$. As $h(z) \in \mathcal{A}^{m}(\Xi)$ is parameter-dependent elliptic, there is an $h^{-1}(z) \in \mathcal{M}^{-m}(\Xi)$ such that $h^{-1}(z) h(z)=h(z) h^{-1}(z)=1$ for all $z \in \Xi$ (cf. [Sch98, 1.2.4]). By Proposition 1.3, we have $h^{-1}(z) a_{s}(z) \in \mathcal{M}^{-\infty}(\Xi)$. Hence the operator $1+h^{-1}(z) a_{s}(z)$ is invertible for all but countably many $z \in \Xi$ and its inverse is of the form $1+g(z)$ with $g(z) \in \mathcal{M}^{-\infty}(\Xi)$ (cf. Lemma 4.3 .13 in [SS94]). Now it is easy to check that $a^{-1}(z)=(1+g(z)) h^{-1}(z)$ fills the bill.

The operator $a^{-1}(z)$ is called the resolvent of $a(z)$. Evidently, it is parameter-dependent elliptic along with $a(z)$. In Section 4 we show an explicit formula for the principal part of $a^{-1}(z)$.

\section{Characteristic values}

In the sequel, an important role is played by the notion of the multiplicity of a characteristic value of a meromorphic operator-valued function. This concept goes as far as Gokhberg and Sigal [GS71] who extended the work of Krein and Trofimov [KT69] for analytic operator-valued functions. 
Let $a(z) \in \mathcal{M}^{m}(\Xi)$ be a meromorphic function in the strip $\Xi$ with values in $\Psi_{\mathrm{cl}}^{m}(X)$. Our standing assumption on $a(z)$ is that this function is parameter-dependent elliptic, as is explained in Section 1.

For a fixed $z \in \Xi$ away from the set of poles, $a(z)$ can be thought of as an operator $H^{s}(X) \rightarrow H^{s-m}(X)$ for any one $s \in \mathbb{R}$. The particular choice of $s$ is actually not important because the kernel and the cokernel of $a(z)$ consist of $C^{\infty}$ functions on $X$.

A point $z_{0} \in \Xi$ is said to be a characteristic value of $a(z)$ if there exists a holomorphic function $u(z)$ in a neighbourhood of $z_{0}$ with values in $H^{s}(X)$, such that $u\left(z_{0}\right) \neq 0$ but $a(z) u(z)$ is holomorphic at $z_{0}$ and vanishes at this point. It is worth pointing out that $a(z) u(z)$ is not a priori defined at $z_{0}$, however, it is well-defined in a punctured neighbourhood of $z_{0}$. We call $u(z)$ a root function of $a(z)$ at $z_{0}$.

Suppose $z_{0}$ is a characteristic value of $a(z)$ and $u(z)$ is a corresponding root function. The order of $z_{0}$ as a zero of $a(z) u(z)$ is called the multiplicity of $u(z)$, and the function $u\left(z_{0}\right) \in H^{s}(X)$ an eigenfunction of $a(z)$ at $z_{0}$. If supplemented by the zero function on $X$, the eigenfunctions of $a(z)$ at $z_{0}$ form a linear space. This space is called the kernel of $a(z)$ at $z_{0}$, and is denoted by $\operatorname{ker} a\left(z_{0}\right)$. By the rank of an eigenfunction $u_{0} \in H^{s}(X)$ we mean the supremum of the multiplicities of all root functions $u(z)$ such that $u\left(z_{0}\right)=u_{0}$.

Proposition 2.1 For any characteristic value $z_{0}$ of $a(z)$, the kernel of $a(z)$ at $z_{0}$ is finite-dimensional and consists of $C^{\infty}$ functions on X. Moreover, the rank of each eigenfunction of $a(z)$ at $z_{0}$ is finite.

Proof. We have

$$
a(z)=\sum_{j=-\mu}^{-1} a_{j}\left(z-z_{0}\right)^{j}+h(z)
$$

in a neighbourhood of $z_{0}$, where $a_{j}$ are smoothing operators of finite rank on $X$ and $h(z)$ is a holomorphic function near $z_{0}$ with values in $\Psi_{\mathrm{cl}}^{m}(X)$.

Let us observe from the very beginning that $h\left(z_{0}\right)$ is an elliptic pseudodifferential operator on $X$. Indeed, write $a(z)=a_{s}(z)+a_{r}(z)$ by Proposition 1.2 , where

$$
\begin{aligned}
& a_{s}(z) \in \mathcal{M}^{-\infty}(\Xi), \\
& a_{r}(z) \in \mathcal{A}^{m}(\Xi) .
\end{aligned}
$$

Comparing this with (2.1) near $z_{0}$, we see that $\sigma^{m}\left(h\left(z_{0}\right)\right)=\sigma^{m}\left(a_{r}\left(z_{0}\right)\right.$. As $a_{r}\left(z_{0}\right)$ is elliptic, so is $h\left(z_{0}\right)$, which is our claim.

If $u(z)$ is a holomorphic function in a neighbourhood of $z_{0}$ with values in $H^{s}(X)$, then

$$
a(z) u(z)=\sum_{\nu=-\mu}^{0}\left(\sum_{j+k=\nu} \frac{1}{k !} a_{j} u^{(k)}\left(z_{0}\right)\right)\left(z-z_{0}\right)^{\nu}+h\left(z_{0}\right) u\left(z_{0}\right)+O\left(\left|z-z_{0}\right|\right)
$$


close to $z_{0}$. Hence it follows that in order that $u(z)$ be a root function of $a(z)$ at $z_{0}$ it is necessary and sufficient that $u\left(z_{0}\right) \neq 0$ and

$$
\begin{aligned}
\sum_{k=0}^{\mu+\nu} \frac{1}{k !} a_{\nu-k} u^{(k)}\left(z_{0}\right) & =0 \quad \text { for all } \nu=-\mu, \ldots,-1 ; \\
h\left(z_{0}\right) u\left(z_{0}\right) & =-\sum_{k=1}^{\mu} \frac{1}{k !} a_{-k} u^{(k)}\left(z_{0}\right)
\end{aligned}
$$

Since $h\left(z_{0}\right)$ is an elliptic operator in $\Psi_{\mathrm{cl}}^{m}(X)$, the second equation of (2.2) shows that $u\left(z_{0}\right)$ lies in a finite-dimensional subspace of $C^{\infty}(X)$ which is completely determined by $h\left(z_{0}\right)$ and the operators $a_{-\mu}, \ldots, a_{-1}$ in (2.1). This establishes the first part of the proposition.

To prove the second part, let $u(z)$ be a root function of $a(z)$ at $z_{0}$. This means that $f(z)=a(z) u(z)$ is a holomorphic function near $z_{0}$ and $f\left(z_{0}\right)=0$. By Proposition 1.4, we get $u(z)=a^{-1}(z) f(z)$ in a punctured neighbourhood of $z_{0}$. As $a^{-1}(z) \in \mathcal{M}^{-m}(\Xi)$ and $u\left(z_{0}\right) \neq 0$, we can assert that the order of $z_{0}$ as a zero of $f(z)$ does not exceed the order of $z_{0}$ as a pole of $a^{-1}(z)$. This latter is finite, which completes the proof.

By a canonical system of eigenfunctions of $a(z)$ at $z_{0}$ we mean any system of eigenfunctions $u_{0}^{(1)}, \ldots, u_{0}^{(I)}$ with the property that the rank of $u_{0}^{(1)}$ is the maximum of the ranks of all eigenfunctions of $a(z)$ at $z_{0}$ and the rank of $u_{0}^{(i)}$ is the maximum of the ranks of all eigenfunctions in a direct complement in ker $a\left(z_{0}\right)$ of the linear span of the vectors $u_{0}^{(1)}, \ldots, u_{0}^{(i-1)}$, for $i=2, \ldots, I$. Let $r_{i}$ be the rank of $u_{0}^{(i)}$, for $i=1, \ldots, I$. It is a simple matter to see that the rank of any eigenfunction of $a(z)$ at the characteristic value $z_{0}$ is always equal to one of the $r_{i}$. Hence it follows that the numbers $r_{i}$ are determined uniquely by the function $a(z)$. Note that a canonical system of eigenfunctions is not, in general, uniquely determined. The numbers $r_{i}$ are said to be partial null multiplicities of the characteristic value $z_{0}$ of $a(z)$. Following [GS71], we call $\mathfrak{n}\left(a\left(z_{0}\right)\right)=r_{1}+\ldots+r_{I}$ the null multiplicity of the characteristic value $z_{0}$ of $a(z)$. If $a(z)$ has no root function at $z_{0}$, we set $\mathfrak{n}\left(a\left(z_{0}\right)\right)=0$.

We may apply these arguments as well to the inverse family $a^{-1}(z)$, as is clear from Proposition 1.4. By abuse of notation, we call both the characteristic values of $a(z)$ and those of $a^{-1}(z)$ the singular values of $a(z)$. Suppose that $z_{0}$ is a characteristic value of $a^{-1}(z)$ in the strip $\Xi$. Denote by $\varrho_{1}, \ldots, \varrho_{J}$ the partial null multiplicities of this characteristic value of $a^{-1}(z)$. The numbers $\varrho_{\iota}$ are also referred to as the partial polar multiplicities of the singular value $z_{0}$ of $a(z)$. Moreover, we call $\mathfrak{n}\left(a^{-1}\left(z_{0}\right)\right)=\varrho_{1}+\ldots+\varrho_{J}$ the polar multiplicity of the singular value $z_{0}$ of $a(z)$ and denote it by $\mathfrak{p}\left(a\left(z_{0}\right)\right)$ (cf. [GS71]). If $a^{-1}(z)$ has no root function at $z_{0}$, we set $\mathfrak{p}\left(a\left(z_{0}\right)\right)=0$.

Definition 2.2 The quantity $\mathfrak{m}\left(a\left(z_{0}\right)\right)=\mathfrak{n}\left(a\left(z_{0}\right)\right)-\mathfrak{p}\left(a\left(z_{0}\right)\right)$ is called the multiplicity of a singular value $z_{0}$ of the family a $(z)$. 
If $a(z)$ is holomorphic at a point $z_{0} \in \Xi$ and the operator $a\left(z_{0}\right)$ is invertible, then $z_{0}$ is said to be a regular point of $a(z)$. Note that the multiplicity of each regular point of $a(z)$ is equal to zero.

We will need an auxiliary result concerning the multiplicity of a characteristic value.

Proposition 2.3 Assume that $z_{0} \in \Xi$ is a characteristic value of $a(z) \in$ $\mathcal{M}^{m}(\Xi)$. If $b_{j}(z), j=1,2$, are invertible holomorphic functions near $z_{0}$ with values in $\Psi_{\mathrm{cl}}^{n_{j}}(X)$, then $z_{0}$ is a characteristic value of $c(z)=b_{2}(z) a(z) b_{1}(z)$ and the partial null multiplicities of $z_{0}$ for $c(z)$ and $a(z)$ coincide.

Proof. Indeed, the multiplicity of any root function $u(z)$ of $a(z)$ at $z_{0}$ is equal to the multiplicity of the root function $b_{1}^{-1}(z) u(z)$ of $c(z)$ at $z_{0}$. In particular, the kernels of $a(z)$ and $c(z)$ at $z_{0}$ are isomorphic, and the desired conclusion follows.

Proposition 2.3 actually shows that both the partial null multiplicities and the partial polar multiplicities of the singular value $z_{0}$ for $c(z)$ and $a(z)$ coincide. In particular, we get $\mathfrak{m}\left(c\left(z_{0}\right)\right)=\mathfrak{m}\left(a\left(z_{0}\right)\right)$.

\section{Factorisation}

In this section we briefly sketch a special factorisation of a meromorphic operator-valued function close to a characteristic value, as is given by Gokhberg and Sigal [GS71].

Proposition 3.1 Let $a(z) \in \mathcal{M}^{m}(\Xi)$ be parameter-dependent elliptic and $z_{0} \in \Xi$ be a singular value of $a(z)$. Then there are invertible holomorphic functions $b_{1}(z)$ and $b_{2}(z)$ near $z_{0}$ with values in $\Psi_{\mathrm{cl}}^{-m}(X)$ and $\Psi_{\mathrm{cl}}^{0}(X)$, respectively, such that

$$
b_{2}(z) a(z) b_{1}(z)=\pi_{0}+\sum_{\nu=1}^{N} \pi_{\nu}\left(z-z_{0}\right)^{m_{\nu}}
$$

close to $z_{0}$, where $m_{1} \leq \ldots \leq m_{N}$ are integers and $\pi_{0}, \pi_{1}, \ldots, \pi_{N}$ are mutually orthogonal projections, such that $\pi_{1}, \ldots, \pi_{N} \in \Psi^{-\infty}(X)$ are of rank 1 and $\pi_{0}+\sum_{\nu=1}^{N} \pi_{\nu}=1$.

Proof. The proof consists in an inspection of the proof of Theorem 3.1 in [GS71]. For the convenience of the reader we repeat the relevant material from [GS71] with necessary modifications.

Let us expand $a(z)$ as a Laurent series (2.1) in a neighbourhood $O$ of the point $z_{0}$. By the above, $h\left(z_{0}\right)$ is an elliptic pseudodifferential operator of order $m$ on $X$. As $\sigma^{m}(h(z))=\sigma^{m}(a(z))$ for $z$ in $O \backslash\left\{z_{0}\right\}$ and $a(z)$ is invertible in a punctured neighbourhood of $z_{0}$, it follows that the index of the operator $h\left(z_{0}\right)$ is equal to 0 . We can therefore assert that there is a smoothing operator $s_{0}$ of finite rank on $X$, such that $e_{0}=s_{0}+h\left(z_{0}\right)$ is invertible. By continuity, the operator $e(z)=s_{0}+h(z)$ is invertible in some 
neighbourhood $O^{\prime}$ of $z_{0}$. By shrinking $O$, if necessary, we may assume that $O_{1}=O$. Then we get

$$
\begin{aligned}
a(z) & =g(z)+e(z) \\
& =e(z)\left(1+e^{-1}(z) g(z)\right),
\end{aligned}
$$

for all $z \in O$, where $g(z)=\sum_{j=-\mu}^{-1} a_{j}\left(z-z_{0}\right)^{j}-s_{0}$.

Clearly, $s(z)=e^{-1}(z) g(z)$ is a holomorphic function in $O \backslash\left\{z_{0}\right\}$ whose values are smoothing operators of finite rank on $X$. In the neighbourhood $O$ it admits a representation

$$
s(z)=\sum_{j=-\mu}^{-1} s_{j}\left(z-z_{0}\right)^{j}+t(z)
$$

where $s_{-\mu}, \ldots, s_{-1}$ are smoothing operators of finite rank on $X$ and $t(z)$ is a holomorphic function in $O$ with values in smoothing operators of finite rank on $X$.

Let $\mathcal{N}$ denote the intersection of the null-spaces of the operators $a_{j}$, $j=-\mu, \ldots,-1$, and $s_{0}$ in $\mathcal{D}^{\prime}(X)$. Since all these operators are of finite rank, we see that $\mathcal{N}$ is a subspace of $\mathcal{D}^{\prime}(X)$ of finite codimension. If $u \in \mathcal{N}$, then $g(z) u=0$, and so $s(z) u=0$ for all $z \in O$.

In $\mathcal{N}$, we consider the subspace $\mathcal{N}_{0}$ consisting of all functions $u \in \mathcal{N}$ satisfying $s_{-\mu} u=\ldots=s_{-1} u=0$. This subspace has a finite codimension in $\mathcal{N}$ and hence in $\mathcal{D}^{\prime}(X)$. A familiar argument shows that there exists a direct complement $\mathcal{D}^{\prime}(X) \ominus \mathcal{N}_{0}$ of $\mathcal{N}_{0}$ in $\mathcal{D}^{\prime}(X)$ which is invariant with respect to each of the operators $s_{-\mu}, \ldots, s_{-1}$ (as well as $a_{-\mu}, \ldots, a_{-1}$ and $s_{0}$, but we will not use this latter fact). Moreover, since all the $a_{j}, s_{0}$ and $s_{j}$ are smoothing operators, it follows that $\mathcal{D}^{\prime}(X) \ominus \mathcal{N}_{0}$ is a subspace of $C^{\infty}(X)$.

Let $\pi$ be the projection which projects $\mathcal{D}^{\prime}(X)$ onto $\mathcal{D}^{\prime}(X) \ominus \mathcal{N}_{0}$ parallel to $\mathcal{N}_{0}$. By the above, $\pi$ is a smoothing operator. Set $\pi_{0}=1-\pi$.

It is a simple matter to see that $\pi_{0} s(z) \pi=\pi_{0} t(z) \pi$. From this we deduce that

$$
\begin{aligned}
1+s(z) & =1+\pi s(z) \pi+\pi_{0} t(z) \pi \\
& =(1+\pi s(z) \pi)\left(1+\pi_{0} t(z) \pi\right) .
\end{aligned}
$$

The operator-valued function $f(z)=1+\pi_{0} t(z) \pi$ is holomorphic in $O$, and its values are invertible operators, namely $f^{-1}(z)=1-\pi_{0} t(z) \pi$. Thus $a(z)$ can be represented in the form $a(z)=e(z) d(z) f(z)$, with $d(z)$ given by $d(z)=1+\pi s(z) \pi$. For $z \in O$, the operator $\pi d(z) \pi$ can be regarded as acting in the finite-dimensional space $\pi \mathcal{D}^{\prime}(X)$. By an argument of [GS71, $1.3]$, this operator can be represented in the form $\pi d(z) \pi=\epsilon_{1}(z) \tilde{c}(z) f_{1}(z)$, where $e_{1}(z)$ and $f_{1}(z)$ are holomorphic functions in $O$ taking their values in the group of invertible linear operators in $\pi \mathcal{D}^{\prime}(X)$, and $\tilde{c}(z)$ is of the form $\tilde{c}(z)=\sum_{\nu=1}^{N} \tilde{\pi}_{\nu}\left(z-z_{0}\right)^{m_{\nu}}$. Here, $m_{1} \leq \ldots \leq m_{N}$ are integer numbers, 
$\tilde{\pi}_{1}, \ldots, \tilde{\pi}_{N}$ are pairwise orthogonal projections acting in the space $\pi \mathcal{D}^{\prime}(X)$. and $N$ is the rank of the projection $\sum_{\nu=1}^{N} \tilde{\pi}_{\nu}$ in $\pi \mathcal{D}^{\prime}(X)$.

It is easy to verify that

$$
d(z)=\left(\pi_{0}+e_{1}(z) \pi\right)\left(\pi_{0}+\sum_{\nu=1}^{N} \pi_{\nu}\left(z-z_{0}\right)^{m_{\nu}}\right)\left(\pi_{0}+f_{1}(z) \pi\right),
$$

where $\pi_{\nu}=\tilde{\pi}_{\nu} \pi, \nu=1, \ldots, N$. Introducing the notation

$$
\begin{aligned}
& b_{1}(z)=f^{-1}(z)\left(\pi_{0}+f_{1}^{-1}(z) \pi\right) \\
& b_{2}(z)=\left(\pi_{0}+e_{1}^{-1}(z) \pi\right) e^{-1}(z)
\end{aligned}
$$

we obtain the representation (3.1). Finally, as $a(z)$ is invertible at points close to $z_{0}$, so is $b_{2}(z) a(z) b_{1}(z)$. Hence it follows that $\pi_{0}+\sum_{\nu=1}^{N} \pi_{\nu}=1$, which completes the proof.

Following [GS71], we call (3.1) a normal factorisation of $a(z)$ at the point $z_{0}$. The principal significance of such a factorisation is that it allows one to highlight the structure of the inverse operator-valued function. Namely, if $b_{2}(z) a(z) b_{1}(z)=c(z)$ near $z_{0}$, with $c(z)$ given by the right-hand side of $(3.1)$, then $a^{-1}(z)=b_{1}(z) c^{-1}(z) b_{2}(z)$ in a punctured neighbourhood of $z_{0}$, where

$$
c^{-1}(z)=\pi_{0}+\sum_{\nu=1}^{N} \pi_{\nu}\left(z-z_{0}\right)^{-m_{\nu}}
$$

On the other hand, if having a normal factorisation of $a(z)$ at $z_{0}$, we can show explicitly the partial null and polar multiplicities of the singular value $z_{0}$ of $a(z)$. Namely, suppose that the numbers $m_{\nu}, \nu=1, \ldots, N$, from (3.1) satisfy the conditions

$$
\begin{gathered}
m_{1} \leq \cdots \leq m_{J}<0, \\
m_{J+1} \leq \cdots \leq m_{N-I}=0, \\
m_{N-I+1} \leq \cdots \leq m_{N}>0
\end{gathered}
$$

where $0 \leq I \leq N$ and $0 \leq J \leq N-I$. Then, the partial null multiplicities of the singular value $z_{0}$ of $a(z)$ are equal to $m_{N-I+1}, \ldots m_{N}$, the partial polar multiplicities of the singular value $z_{0}$ of $a(z)$ are equal to $m_{1}, \ldots m_{J}$, whence $\mathfrak{m}\left(a\left(z_{0}\right)\right)=\sum_{\nu=1}^{N} m_{\nu}$. We also deduce that the maximum of the ranks of all eigenvectors of $a(z)$ corresponding to a characteristic value $z_{0}$ which is a normal point of $a(z)$ is equal to the order of the pole of $a^{-1}(z)$ at $z_{0}$.

For $a(z) \in \mathcal{M}^{m}(\Xi)$, we denote by p.p. $a(z)$ the principal part of the Laurent expansion of $a(z)$ in a neighbourhood of a singular value $z_{0}$. By definition, p.p. $a(z)$ is a smoothing operator of finite rank on $X$ for all $z$ in a punctured neighbourhood of $z_{0}$. Hence the trace (denoted tr) of p.p. $a(z)$ is well-defined. 
Corollary 3.2 Suppose $a(z) \in \mathcal{M}^{m}(\Xi)$ is parameter-dependent elliptic and $z_{0} \in \Xi$ is a singular value of $a(z)$. Then

$$
\operatorname{tr} \text { p.p. } a^{\prime}(z) a^{-1}(z)=\frac{\mathfrak{m}\left(a\left(z_{0}\right)\right)}{z-z_{0}} .
$$

Proof. Indeed, applying (3.1) yields

$$
\operatorname{tr} \text { p.p. } a^{\prime}(z) a^{-1}(z)=\frac{\mathfrak{m}\left(a\left(z_{0}\right)\right)}{z-z_{0}}-\operatorname{tr} \text { p.p. }\left(b_{1}^{\prime}(z) b_{1}^{-1}(z)+b_{2}^{\prime}(z) b_{2}^{-1}(z)\right)
$$

in a neighbourhood of $z_{0}$. Since both $b_{1}(z)$ and $b_{2}(z)$ are holomorphic and invertible near $z_{0}$, we conclude that

$$
\begin{aligned}
& \text { p.p. } b_{1}^{\prime}(z) b_{1}^{-1}(z)=0, \\
& \text { p.p. } b_{2}^{\prime}(z) b_{2}^{-1}(z)=0,
\end{aligned}
$$

which completes the proof.

In case $a(z)$ is a polynomial operator-valued function this corollary goes back at least as far as Keldysh [Kel51]. The general case is due to Gokhberg and Sigal [GS71].

Given $a(z) \in \mathcal{M}^{m}(\Xi)$, we write $a^{\prime}(z)$ for the function $z \mapsto(a(z))^{\prime}$, the prime meaning the transposed pseudodifferential operator. It is clear that $a^{\prime}(z) \in \mathcal{M}^{m}(\Xi)$; moreover, $a^{\prime}(z)$ is parameter-dependent elliptic if $a(z)$ is.

Corollary 3.3 If $a(z) \in \mathcal{M}^{m}(\Xi)$ is parameter-dependent elliptic, then $a(z)$ and $a^{\prime}(z)$ have the same singular values with the same partial null and polar multiplicities. In particular, $\mathfrak{m}\left(a^{\prime}\left(z_{0}\right)=\mathfrak{m}\left(a\left(z_{0}\right)\right)\right.$.

Proof. This follows immediately from Propositions 2.3 and 3.1 (see also Theorem 5.3 in [GS71]).

\section{Resolvent}

In this section we rehearse the expansion of the principal part of the resolvent $a^{-1}(z)$ from $[\mathrm{GS} 71]$.

Let $a(z) \in \mathcal{M}^{m}(\Xi)$. We assume that $a(z)$ is parameter-dependent elliptic.

Suppose that $z_{0} \in \Xi$ is a characteristic value of $a(z)$ and that $u(z)$ is a root function of $a(z)$ at $z_{0}$. Recall that the value $u\left(z_{0}\right) \in C^{\infty}(X)$ is called an eigenfunction of $a(z)$ at $z_{0}$. Denote by $r$ the multiplicity of $u(z)$. The derivatives

$$
\frac{1}{k !} u^{(k)}\left(z_{0}\right), \quad k=1, \ldots, r-1,
$$

are said to be associated functions for the eigenfunction $u\left(z_{0}\right)$ (a priori they are in a space $\left.H^{s}(X)\right)$. 
Proposition 4.1 For each characteristic value $z_{0}$ of $a(z)$, the associated functions of $a(z)$ at $z_{0}$ lie in a finite-dimensional subspace of $C^{\infty}(X)$.

Proof. We argue as in the proof of Proposition 2.1. Pick a root function $u(z)$ of $a(z)$ at $z_{0}$. Write

$$
\begin{aligned}
& a(z)=\sum_{j=-\mu}^{\infty} a_{j}\left(z-z_{0}\right)^{j}, \\
& u(z)=\sum_{k=0}^{\infty} u_{k}\left(z-z_{0}\right)^{k}
\end{aligned}
$$

in a neighbourhood of $z_{0}$. An easy verification shows that for $u(z)$ to be of multiplicity $r \geq 1$ it is necessary and sufficient that

$$
\begin{aligned}
\sum_{k=0}^{\mu+\nu} a_{\nu-k} u_{k} & =0 & \text { for all } \nu & =-\mu, \ldots,-1 ; \\
a_{0} u_{\nu}+\sum_{k=0}^{\nu-1} a_{\nu-k} u_{k} & =-\sum_{k=\nu+1}^{\mu+\nu} a_{\nu-k} u_{k} & & \text { for all } \nu=0, \ldots, r-1
\end{aligned}
$$

(cf. (2.2)). As $a_{0}$ is an elliptic operator in $\Psi_{\mathrm{cl}}^{m}(X)$ and $a_{-1}, \ldots, a_{-\mu}$ are smoothing operators of finite rank on $X$, we deduce by induction from the second group of equalities (4.1) that each function $u_{k}, k=0,1, \ldots, r-1$, belongs to a subspace of $C^{\infty}(X)$ of finite dimension. Moreover, this subspace is completely determined by the operators $a_{-\mu}, \ldots, a_{k}$, which is precisely our assertion.

If $u_{1}, \ldots, u_{r-1}$ are associated functions for an eigenfunction $u_{0}$ of $a(z)$ at $z_{0}$, then any system $u_{0}, u_{1}, \ldots, u_{N}$ with $\nu \leq r-1$ is called a chain consisting of an eigenfunction and associated functions of $a(z)$ at $z_{0}$.

It is easy to see that a system $u_{0}, u_{1}, \ldots, u_{N}$ of functions in $H^{s}(X)$ forms a chain if and only if there are functions $u_{N+1}, \ldots, u_{N+\mu} \in H^{s}(X)$ such that

$$
\sum_{k=0}^{\mu+\nu} a_{\nu-k} u_{k}=0 \quad \text { for } \quad \nu=-\mu, \ldots, N
$$

(cf. (4.1)).

Let $u_{0}^{(1)}, \ldots, u_{0}^{(I)}$ be a canonical system of eigenfunctions of $a(z)$ at $z_{0}$, $I$ being the dimension of $\operatorname{ker} a\left(z_{0}\right)$. Denote by $r_{i}$ the rank of $u_{0}^{(i)}$. If, for each $i=1, \ldots, I$, the functions $u_{0}^{(i)}, u_{1}^{(i)}, \ldots, u_{r_{i}-1}^{(i)}$ form a chain consisting of an eigenfunction and associated functions of $a(z)$ at $z_{0}$, then the system

$$
\left(u_{0}^{(i)}, u_{1}^{(i)}, \ldots, u_{r_{i}-1}^{(i)}\right)_{i=1, \ldots, I}
$$

is called a canonical system of eigenfunctions and associated functions of $a(z)$ at $z_{0}$.

The following result will be needed below. It is proved by Gokhberg and Sigal [GS71] for meromorphic operator-valued functions. They refer to Keldysh [Kel51] for the case of polynomials with values in operators on a Hilbert space. 
Proposition 4.2 For each characteristic value $z_{0}$ of $a(z)$, there are canonical systems

$$
\begin{aligned}
& \left(u_{0}^{(i)}, u_{1}^{(i)}, \ldots, u_{r_{i}-1}^{(i)}\right)_{i=1, \ldots, I}, \\
& \left(g_{0}^{(i)}, g_{1}^{(i)}, \ldots, g_{r_{i}-1}^{(i)}\right)_{i=1, \ldots, I}
\end{aligned}
$$

of eigenfunctions and associated functions of $a(z)$ and $a^{\prime}(z)$ at $z_{0}$, respectively, such that

$$
\text { p.p. } a^{-1}(z)=\sum_{i=1}^{I} \sum_{j=-r_{i}}^{-1}\left(z-z_{0}\right)^{j} \sum_{k=0}^{r_{i}+j}\left\langle g_{k}^{(i)}, \cdot\right\rangle u_{r_{i}+j-k}^{(i)} \text {. }
$$

Proof. Cf. Theorem 7.1 in [GS71].

\section{Unitary reduction}

We now turn to pseudodifferential equations on a cylinder $\mathcal{C}=\mathbb{R} \times X$ over a $C^{\infty}$ compact closed manifold $X$.

Any function on $\mathcal{C}$ may be thought of as a function on $\mathbb{R}$ with values in a function space on $X$. In particular, we write $\mathcal{S}(\mathcal{C})=\mathcal{S}(\mathbb{R}) \otimes_{\pi} C^{\infty}(X)$ for the space of rapidly decreasing functions on the real axis with values in $C^{\infty}(X)$. If $u \in e^{-\gamma t} \mathcal{S}(\mathcal{C})$, where $\gamma \in \mathbb{R}$, then the Fourier transform $\mathcal{F} u(z)=\mathcal{F}_{t \mapsto \Re z}\left(e^{\Im z t} u\right)$ of $u$ is well-defined for all $z$ lying on the horizontal line $\Gamma_{\gamma}=\left\{z \in \mathbb{C}: \Im_{z} z=\gamma\right\}$. Moreover, $\mathcal{F} u(\tau+i \gamma)$ is a rapidly decreasing function of $\tau \in \mathbb{R}$ with respect to each seminorm in $C^{\infty}(X)$.

Let $a(z) \in \mathcal{M}^{m}(\Xi)$. Pick a $\gamma \in(a, b)$ such that the line $\Gamma_{\gamma}$ is free from the poles of $a(z)$. For each $u \in e^{-\gamma t} \mathcal{S}(\mathcal{C})$, the integral

$$
\begin{aligned}
A u(t) & =\frac{1}{2 \pi} \int_{\Gamma_{\gamma}} e^{i t z} a(z) \mathcal{F} u(z) d z \\
& =e^{-\gamma t} \mathcal{F}_{\tau \mapsto t}^{-1} a(\tau+i \gamma) \mathcal{F}_{t \mapsto \tau}\left(e^{\gamma t} u\right), \quad t \in \mathbb{R},
\end{aligned}
$$

gives a rapidly decreasing function on $\mathbb{R}$ with values in $C^{\infty}(X)$, modulo the factor $e^{-\gamma t}$. In fact, $A$ is a continuous mapping of $e^{-\gamma t} \mathcal{S}(\mathcal{C}) \rightarrow e^{-\gamma t} \mathcal{S}(\mathcal{C})$, as is easy to see.

Lemma 5.1 Suppose $u \in e^{-\gamma t} \mathcal{S}(\mathcal{C})$. Then $\mathcal{F}(A u)(z)=a(z) \mathcal{F} u(z)$ for all $z \in \Gamma_{\gamma}$.

Proof. Using the equality $\mathcal{F} u(\tau+i \gamma)=\mathcal{F}_{t \mapsto \tau}\left(e^{\gamma t} u\right)$ for $\tau \in \mathbb{R}$, we obtain

$$
\begin{aligned}
\mathcal{F}(A u)(\tau+i \gamma) & =\mathcal{F}_{t \mapsto \tau}\left(e^{\gamma t} A u\right) \\
& =a(\tau+i \gamma) \mathcal{F}_{t \mapsto \tau}\left(e^{\gamma t} u\right) \\
& =a(\tau+i \gamma) \mathcal{F} u(\tau+i \gamma),
\end{aligned}
$$


the second equality being due to (5.1) and the fact that $a(\tau+i \gamma) \mathcal{F}_{t \mapsto \tau}\left(e^{\gamma t} u\right)$ is a rapidly decreasing function of $\tau \in \mathbb{R}$ with values in $C^{\infty}(X)$. This is the desired conclusion.

Roughly speaking, Lemma 5.1 just amounts to saying that the Fourier transform of the temperate distribution $u(t) \equiv 1$ is a constant multiple of the Dirac delta-function. In fact, $\int_{\mathbb{R}} e^{-i \tau t} d t=2 \pi \delta(\tau)$ for each $\tau \in \mathbb{R}$.

Our next goal is to extend $A$ to a continuous mapping of weighted Sobolev spaces on the cylinder, $H^{s, \gamma}(\mathcal{C})$. For $s \in \mathbb{Z}_{+}$and $\gamma \in \mathbb{R}$, we mean by $H^{s, \gamma}(\mathcal{C})$ the completion of $C_{\text {comp }}^{\infty}(\mathcal{C})$ with respect to the norm

$$
\|u\|_{H^{s, \gamma}(\mathcal{C})}=\left(\int_{\mathbb{R}} \sum_{j+A \leq s}\left\|D^{j}\left(e^{\gamma t} u\right)\right\|_{H^{A}(X)}^{2} d t\right)^{1 / 2} .
$$

Obviously, $H^{s, \gamma}(\mathcal{C})$ is a Hilbert space; however, the Hilbert structure is not canonical. If $s$ is a negative integer, we set $H^{s, \gamma}(\mathcal{C})$ to be the dual of $H^{-s,-\gamma}(\mathcal{C})$. For fractional $s$, the space $H^{s, \gamma}(\mathcal{C})$ is defined by (complex) interpolation.

Proposition 5.2 As defined by (5.1), A extends to a continuous mapping $H^{s, \gamma}(\mathcal{C}) \rightarrow H^{s-m, \gamma}(\mathcal{C})$ for each $s \in \mathbb{R}$.

Proof. Fix a family of order reductions $\Lambda^{s}(\tau) \in \Psi_{\text {cl }}^{s}(X ; \mathbb{R}), s \in \mathbb{R}$, on $X$. Then

$$
\|u\|_{H^{s, \gamma}(\mathcal{C})} \sim\left(\int_{\Gamma_{\gamma}}\left\|\Lambda^{s}(\Re z) \mathcal{F} u(z)\right\|_{L^{2}(X)}^{2} d z\right)^{1 / 2},
$$

the equivalence of two norms meaning that their ratio is bounded both above and below by positive constants independent of $u$. Hence

$$
\begin{aligned}
\|A u\|_{H^{s-m, \gamma}(\mathcal{C})} & \sim\left(\int_{\Gamma_{\gamma}}\left\|\Lambda^{s-m}(\Re z) a(z) \mathcal{F} u(z)\right\|_{L^{2}(X)}^{2} d z\right)^{1 / 2} \\
& \leq c\left(\sup _{\tau \in \mathbb{R}}\left\|\Lambda^{s-m}(\tau) a(\tau+i \gamma) \Lambda^{-s}(\tau)\right\|_{\mathcal{L}\left(L^{2}(X)\right)}\right)\|u\|_{H^{s, \gamma}(\mathcal{C})}
\end{aligned}
$$

for all $u \in C_{\text {comp }}^{\infty}(\mathcal{C})$, the constant $c$ being independent of $A$ and $u$. Since the seminorm

$$
a(\tau) \mapsto \sup _{\tau \in \mathbb{R}}\left\|\Lambda^{s-m}(\tau) a(\tau) \Lambda^{-s}(\tau)\right\|_{\mathcal{L}\left(L^{2}(X)\right)}
$$

is continuous on $\Psi_{c l}^{m}(X ; \mathbb{R})$, the proof is complete.

Given an $f \in H^{s-m, \gamma}(\mathcal{C})$, consider the equation $A u=f$ for an unknown function $u \in H^{s, \gamma}(\mathcal{C})$. The solvability theory of this equation is a direct consequence of the fact that the Fourier transform $u(t) \mapsto \mathcal{F} u(\tau+i \gamma)$ extends to a unitary isomorphism $H^{0, \gamma}(\mathcal{C}) \stackrel{\cong}{\rightrightarrows} L^{2}\left(\Gamma_{\gamma}, L^{2}(X)\right)$, up to an inessential factor $2 \pi$. This reduces the problem to a parameter-dependent equation on the base $X$. 
Proposition 5.3 If the weight line $\Gamma_{\gamma}$ lies away from the set of singular values of $a(z)$, then the mapping $A: H^{s, \gamma}(\mathcal{C}) \rightarrow H^{s-m, \gamma}(\mathcal{C})$ is one-to-one and onto, for every $s \in \mathbb{R}$. Moreover, the inverse mapping is given by the formula

$$
A^{-1} f(t)=\frac{1}{2 \pi} \int_{\Gamma_{\gamma}} e^{i t z} a^{-1}(z) \mathcal{F} f(z) d z, \quad t \in \mathbb{R}
$$

Proof. Indeed, Proposition 1.4 shows that $a^{-1}(z) \in \mathcal{M}^{-m}(\Xi)$. Moreover, the line $\Gamma_{\gamma}$ is free from the poles of $a^{-1}$, which is guaranteed by the assumption. Hence it follows, by Proposition 5.2, that the operator $A^{-1}$ given by (5.3) extends to a continuous mapping $H^{s-m, \gamma}(\mathcal{C}) \rightarrow H^{s, \gamma}(\mathcal{C})$, for any $s \in \mathbb{R}$. It remains to prove that $A^{-1} A=1$ and $A A^{-1}=1$. We restrict our attention to the first equality; the proof of the second one is similar. Obviously, it suffices to show that $A^{-1} A=1$ on $C_{\text {comp }}^{\infty}(\mathcal{C})$ because this subspace is dense in $H^{s, \gamma}(\mathcal{C})$. But for $u \in C_{\text {comp }}^{\infty}(\mathcal{C})$ we may use Lemma 5.1 to obtain

$$
\begin{aligned}
A^{-1} A u(t) & =\frac{1}{2 \pi} \int_{\Gamma_{\gamma}} e^{i t z} a^{-1}(z) a(z) \mathcal{F} u(z) d z \\
& =\frac{1}{2 \pi} \int_{\Gamma_{\gamma}} e^{i t z} \mathcal{F} u(z) d z \\
& =u(t)
\end{aligned}
$$

the latter equality being a consequence of the Fourier inversion formula. This is our claim.

Proposition 5.3 is an underlying technical tool for studying more intricate settings of the problem $A u=f$.

\section{Inhomogeneous equation}

The factor $e^{\gamma t}$ entering into (5.2) can not control independently the behaviour of functions at $t=-\infty$ and $t=\infty$. To do this, we introduce yet another scale of weighted Sobolev spaces on the cylinder, which includes two weight parameters. Namely, let $w=\left(w_{-}, w_{+}\right)$be a pair of real numbers to inspect the growth of functions at $t= \pm \infty$. Fix a cut-off function $\omega$ for the point $t=-\infty$ on the real axis, i.e., $\omega$ is a $C^{\infty}$ function on $\mathbb{R}$ equal to 1 near $t=-\infty$ and vanishing near $t=\infty$. For $s \in \mathbb{R}$, set

$$
H^{s, w}(\mathcal{C})=\omega H^{s,-w}-(\mathcal{C})+(1-\omega) H^{s, w+}(\mathcal{C}),
$$

the right-hand side being understood in the sense of non-direct sum of Fréchet spaces. In particular, taking $w=(-\gamma, \gamma)$ we get $H^{s, w}(\mathcal{C})=H^{s, \gamma}(\mathcal{C})$ for any $s, \gamma \in \mathbb{R}$. 
Lemma 6.1 As defined by $(6.1)$, the space $H^{s, w}(\mathcal{C})$ is equivalently topologised under the norm

$$
\|u\|_{H^{s, w}(\mathcal{C})}=\left(\|\omega u\|_{H^{s,-w}-(\mathcal{C})}^{2}+\|(1-\omega) u\|_{H^{s, w}+(\mathcal{C})}^{2}\right)^{1 / 2} .
$$

Proof. The proof follows from the fact that the spaces $H^{s, \gamma}(\mathcal{C})$ are invariant under multiplication by smooth functions on $\mathbb{R}$ constant in the complement of a compact interval.

It follows that $H^{s, w}(\mathcal{C})$ bears a Hilbert structure. Note that this structure depends on the particular choice of $\omega$ while the space $H^{s, w}(\mathcal{C})$ itself does not. The dual of $H^{s, w}(\mathcal{C})$ is still identified with $H^{-s,-w}(\mathcal{C})$, for each $s$ and $w$.

Lemma 6.2 Let $w=\left(w_{-}, w_{+}\right)$satisfy $-w_{-} \leq w_{+}$. Then, in order that a function $u$ belong to $H^{s, w}(\mathcal{C})$ it is necessary and sufficient that $u \in H^{s, \gamma}(\mathcal{C})$ for each $-w_{-} \leq \gamma \leq w_{+}$.

Proof. To prove the necessity, we make use of the triangle inequality to get

$$
\begin{aligned}
\|u\|_{H^{s, \gamma}(\mathcal{C})}^{2} & \leq 2\left(\|\omega u\|_{H^{s, \gamma}(\mathcal{C})}^{2}+\|(1-\omega) u\|_{H^{s, \gamma}(\mathcal{C})}^{2}\right) \\
& \leq c\|u\|_{H^{s, w}(\mathcal{C})}^{2}
\end{aligned}
$$

for any $u \in C_{\text {comp }}^{\infty}(\mathcal{C})$, with $c$ a constant independent of $u$. The latter inequality follows from Lemma 6.1 and the estimates

$$
\begin{aligned}
\|\omega u\|_{H^{s, \gamma}(\mathcal{C})} & \leq c^{\prime}\|\omega u\|_{H^{s,-w}-(\mathcal{C})}, \\
\|(1-\omega) u\|_{H^{s, \gamma}(\mathcal{C})} & \leq c^{\prime \prime}\|(1-\omega) u\|_{H^{s, w}+(\mathcal{C})}
\end{aligned}
$$

which are due to the condition $-w_{-} \leq \gamma \leq w_{+}$. Conversely, applying Lemma 6.1 implies

$$
\|u\|_{H^{s, w}(\mathcal{C})}^{2} \leq c\left(\|u\|_{H^{s,-w}-(\mathcal{C})}^{2}+\|u\|_{H^{s, w}+(\mathcal{C})}^{2}\right)
$$

for all $u \in C_{\text {comp }}^{\infty}(\mathcal{C})$, the constant $c$ depending only on $\omega$. This proves the sufficiency.

The proof above gives more, namely if $u$ belongs to both $H^{s,-w}-(\mathcal{C})$ and $H^{s, w+}(\mathcal{C})$, then $u \in H^{s, w}(\mathcal{C})$.

When regarded from the point of view of the Fourier transform, the spaces $H^{s, w}(\mathcal{C})$ with $-w_{-} \leq w_{+}$have the following advantage.

Lemma 6.3 Given any $u \in H^{s, w}(\mathcal{C})$, the Fourier transform $\mathcal{F} u(z)$ is holomorphic in the strip $-w_{-}<\Im z<w_{+}$. Moreover, if $j$ is a non-negative integer $\leq s$, then

$$
\left\|z^{j} \mathcal{F} u(z)\right\|_{H^{s-j}(X)} \leq c\left(\frac{1}{\sqrt{w_{-}+\Im z}}+\frac{1}{\sqrt{w_{+}-\Im z}}\right)\|u\|_{H^{s, w}(\mathcal{C})}
$$

for all $z$ in the above strip, with $c>0$ a constant independent of $u$ and $z$. 
Proof. A familiar argument shows that it is sufficient to establish the estimate $(6.2)$ for any function $u \in C_{\text {comp }}^{\infty}(\mathcal{C})$. For this purpose, write $u=\omega u+(1-\omega) u$. We estimate separately the Fourier transforms of $\omega u$ and $(1-\omega) u$. Let $b^{\prime}, b^{\prime \prime} \in \mathbb{R}$ be such that $\omega(t)=1$ for $t \leq b^{\prime}$ and $\omega(t)=0$ for $t \geq b^{\prime \prime}$. Then,

$$
\begin{aligned}
z^{j} \mathcal{F}(\omega u)(z) & =\int_{\mathbb{R}} e^{-i z t} D^{j}(\omega(t) u(t)) d t \\
& =\int_{-\infty}^{b^{\prime \prime}} e^{-i \Re z t} e^{\Im z t} D^{j}(\omega(t) u(t)) d t
\end{aligned}
$$

for all $z \in \mathbb{C}$. Hence it follows, by Hölder's inequality, that

$$
\begin{aligned}
\left\|z^{j} \mathcal{F}(\omega u)(z)\right\|_{H^{A}(X)} & \leq\left(\int_{-\infty}^{b^{\prime \prime}} e^{2\left(w_{-}+\Im z\right) t} d t\right)^{\frac{1}{2}}\left(\int_{\mathbb{R}}\left\|e^{-w_{-} t} D^{j}(\omega u)\right\|_{H^{A}(X)}^{2} d t\right)^{\frac{1}{2}} \\
& \leq c^{\prime} \frac{e^{\left(w_{-}+\Im z\right) b^{\prime \prime}}}{\sqrt{w_{-}+\Im z}}\|\omega u\|_{H^{j+A,-w_{-}}-(\mathcal{C})}
\end{aligned}
$$

for each $z$ in the half-plane $\Im z>-w_{-}$. Here, the constant $c^{\prime}$ depends only on $j$ and $w_{-}$, but not on $u$ and $z$. Analogously,

$$
\left\|z^{j} \mathcal{F}((1-\omega) u)(z)\right\|_{H^{A}(X)} \leq c^{\prime \prime} \frac{e^{\left(-w_{+}+\Im z\right) b^{\prime}}}{\sqrt{w_{+}-\Im z}}\|(1-\omega) u\|_{H^{j+A, w_{+}(\mathcal{C})}}
$$

for any $z$ in the half-plane $\Im z<w_{+}$. the constant $c^{\prime \prime}$ depending only on $j$ and $w_{+}$. Combining these estimates we arrive at (6.2), as desired.

Let $a(z) \in \mathcal{M}^{m}(\Xi)$. Our next objective will be to assign an operator $A: H^{s, w}(\mathcal{C}) \rightarrow H^{s-m, w}(\mathcal{C})$ to $a(z)$, for weight data $w=\left(w_{-}, w_{+}\right)$satisfying $a<-w_{-} \leq w_{+}<b$. Note that formula (5.1) does not fit for the definition of $A$ as the integral on the right side depends on the choice of $\gamma$ in the interval $\left[-w_{-}, w_{+}\right]$. To cope with this difficulty, we shrink the domain of $A$ by considering only those $u \in H^{s, w}(\mathcal{C})$ for which the integral in (5.1) is independent of $\gamma \in\left[-w_{-}, w_{+}\right]$. The following lemma highlights such functions $u$.

Lemma 6.4 Let a $(z)$ have no poles on the lines $\Gamma_{-w_{-}}$and $\Gamma_{w_{+}}$, where $a<-w_{-} \leq w_{+}<b$. Then, for each $u \in H^{s, w}(\mathcal{C})$ with $s=\max (0, m)$, we have

$\int_{\Gamma_{-w_{-}}} e^{i t z} a(z) \mathcal{F} u(z) d z-\int_{\Gamma_{w_{+}}} e^{i t z} a(z) \mathcal{F} u(z) d z=2 \pi i \sum_{\varsigma p \in\left(-w_{-}, w_{+}\right)} \operatorname{res}_{p} e^{i t z} a(z) \mathcal{F} u(z)$.

Proof. Consider a closed contour $l$ which is the boundary of the rectangle with vertices $-T-i w_{-}, T-i w_{-}, T+i w_{+}$and $-T+i w_{+}$(see Fig. 1 ). Choose $T>0$ large enough, so that the rectangle contains all the poles of $a(z)$ in the strip between $\Gamma_{-w_{-}}$and $\Gamma_{w_{+}}$. From Lemma 6.3 we deduce that $F(z)=e^{i t z} a(z) \mathcal{F} u(z)$ is a meromorphic function in the strip 


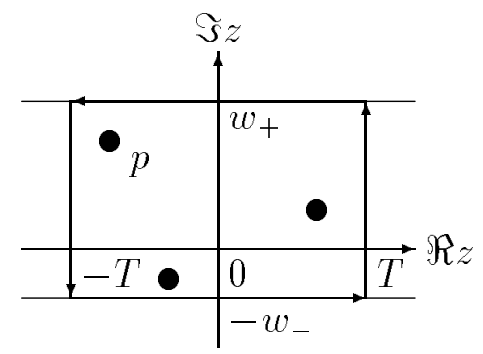

Fig. 1: Auxiliary contour $l$.

$-w_{-}<\Im z<w_{+}$with values in $H^{s-m}(X)$ (for fixed $t \in \mathbb{R}$ ). Hence, the residue formula yields

$$
\begin{aligned}
& \int_{-T-i w_{-}}^{T-i w_{-}} F(z) d z+\int_{T-i w_{-}}^{T+i w_{+}} F(z) d z-\int_{-T+i w_{+}}^{T+i w_{+}} F(z) d z-\int_{-T-i w_{-}}^{-T+i w_{+}} F(z) d z \\
& \quad=2 \pi i \sum_{s p \in\left(-w_{-}, w_{+}\right)} \operatorname{res}_{p} F(z)
\end{aligned}
$$

and we shall have established the lemma if we prove that the integrals $\int_{ \pm T-i w_{-}}^{ \pm T+i w_{+}} F(z) d z$ are infinitesimal with respect to the $H^{-s}(X)$-norm, when $T \rightarrow \infty$. For this purpose, we first make use of Hölder's inequality to obtain

$$
\left\|\int_{ \pm T-i w_{-}}^{ \pm T+i w_{+}} F(z) d z\right\|_{H^{-s}(X)} \leq c\left(\int_{ \pm T-i w_{-}}^{ \pm T+i w_{+}}\|a(z) \mathcal{F} u(z)\|_{H^{-s}(X)}^{2}|d z|\right)^{1 / 2}
$$

where $c=\left(\int_{-w_{-}}^{w_{+}} e^{-2 t \sigma} d \sigma\right)^{1 / 2}$ is independent of $T$. Consider the family of integrals

$$
\int_{ \pm T}^{ \pm \infty}\|a(\tau+i \sigma) \mathcal{F} u(\tau+i \sigma)\|_{H^{-s}(X)}^{2} d \tau
$$

parametrised by $\sigma \in\left(-w_{-}, w_{+}\right)$. Since the operator-valued function $a(z)$ is holomorphic in a half-strip larger than $\pm \Re z>T,-w_{-}<\Im z<w_{+}$, we may invoke the estimates

$$
\|a(\tau+i \sigma)\|_{\mathcal{L}\left(H^{s}(X), H^{s-t}(X)\right)} \leq c \begin{cases}\langle\tau\rangle^{m} & \text { if } t \geq 0 \\ \langle\tau\rangle^{m-t} & \text { if } t \leq 0\end{cases}
$$

for all $z$ therein, where $t \geq m$ is arbitrary real number and $c$ a constant independent of $\pm \tau>T$ and $\sigma \in\left(-w_{-}, w_{+}\right)$(cf. Shubin [Shu87]). Hence it follows that

$$
\begin{aligned}
\int_{ \pm T}^{ \pm \infty}\|a(\tau+i \sigma) \mathcal{F} u(\tau+i \sigma)\|_{H^{-s}(X)}^{2} d \tau & \leq c^{2} \int_{ \pm T}^{ \pm \infty}\langle\tau\rangle^{2 s}\|\mathcal{F} u(\tau+i \sigma)\|_{L^{2}(X)}^{2} d \tau \\
& \leq C\|u\|_{H^{s, \sigma}(\mathcal{C})}^{2},
\end{aligned}
$$

the constant $C$ being independent of $\sigma \in\left(-w_{-}, w_{+}\right)$. On the other hand, the norms $\|u\|_{H^{s, \sigma(\mathcal{C})}}$ are bounded uniformly in $\sigma \in\left(-w_{-}, w_{+}\right)$by the norm 
$\|u\|_{H^{s, w}(\mathcal{C})}$, which is due to Lemma 6.2. We thus conclude that (6.3) is a bounded function on the interval $\sigma \in\left(-w_{-}, w_{+}\right)$. Integrating this function over $\sigma \in\left(-w_{-}, w_{+}\right)$and interchanging the integrals, by Fubini's theorem, we get

$$
\int_{ \pm T}^{ \pm \infty} d \tau \int_{-w_{-}}^{w_{+}}\|a(\tau+i \sigma) \mathcal{F} u(\tau+i \sigma)\|_{H^{-s}(X)}^{2} d \sigma<\infty
$$

Hence it follows that there is a sequence $T_{\nu}>0$ converging to $\infty$, such that

$$
\lim _{\nu \rightarrow \infty} \int_{-w_{-}}^{w_{+}}\left\|a\left( \pm T_{\nu}+i \sigma\right) \mathcal{F} u\left( \pm T_{\nu}+i \sigma\right)\right\|_{H^{-s}(X)}^{2} d \sigma \rightarrow 0,
$$

which is the desired conclusion.

The condition $u \in H^{s, w}(\mathcal{C})$ with $s=\max (0, m)$ might be dropped but we have not been able to do this.

Pick $s \in \mathbb{R}$ with $s \geq \max (0, m)$ and a weight data $w=\left(w_{-}, w_{+}\right)$ satisfying $a<-w_{-} \leq w_{+}<b$. Set

$\operatorname{Dom} A=\left\{u \in H^{s, w}(\mathcal{C}): \operatorname{res}_{p} e^{i t z} a(z) \mathcal{F} u(z)=0\right.$ for $\left.-w_{-}<\Im p<w_{+}\right\}$

where, by abuse of notation, we suppress the dependence of Dom $A$ of $s$ and $w$.

Lemma 6.5 If $a(z)$ is parameter-dependent elliptic, then $\operatorname{Dom} A$ is a closed subspace of finite codimension in $H^{s, w}(\mathcal{C})$. In fact,

$$
\operatorname{codim} \operatorname{Dom} A=\sum_{-w_{-}<\Im p<w_{+}} \mathfrak{p}(a(p)) .
$$

Proof. Indeed, let $p$ be a pole of $a(z)$ in the strip $-w_{-}<\Im z<w_{+}$. From Proposition 3.1 we deduce that $p$ is a characteristic value of the inverse function $a^{-1}(z)$. By Proposition 4.2, there are canonical systems

$$
\begin{aligned}
& \left(f_{0}^{(\iota)}, f_{1}^{(\iota)}, \ldots, f_{\ell_{\iota}-1}^{(\iota)}\right)_{\iota=1, \ldots, J}, \\
& \left(v_{0}^{(\iota)}, v_{1}^{(\iota)}, \ldots, v_{\ell_{\iota}-1}^{(\iota)}\right)_{\iota=1, \ldots, J}
\end{aligned}
$$

of eigenfunctions and associated functions of $a^{-1}(z)$ and $\left(a^{-1}\right)^{\prime}(z)$ at $p$, respectively, such that

$$
\begin{aligned}
\text { p.p. } a(z) & =\text { p.p. }\left(a^{-1}\right)^{-1}(z) \\
& =\sum_{\iota=1}^{J} \sum_{j=-\varrho_{\iota}}^{-1}(z-p)^{j} \sum_{k=0}^{\varrho_{\iota}+j}\left\langle v_{k}^{(\iota)}, \cdot\right\rangle f_{\ell_{\iota}+j-k}^{(\iota)}
\end{aligned}
$$

in a neighbourhood of $p$, the angular brackets standing for a pairing of distributions on $X$. Hence it follows that

$$
\begin{aligned}
\operatorname{res}_{p} & e^{i t z} a(z) \mathcal{F} u(z)=\operatorname{res}_{p} e^{i t z}(\text { p.p. } a(z)) \mathcal{F} u(z) \\
& =\left.\sum_{\iota=1}^{J} \sum_{j=-\varrho_{\iota}}^{-1} \sum_{k=0}^{\varrho_{\iota}+j} \frac{1}{(-j-1) !}\left(\frac{\partial}{\partial z}\right)^{-j-1}\left(e^{i t z} \mathcal{F}_{t \mapsto z}\left\langle v_{k}^{(l)}, u\right\rangle\right)\right|_{z=p} f_{\ell_{\iota}+j-k}^{(\ell)} .
\end{aligned}
$$


The expression on the right-hand side of this equality can be written equivalently as

$$
\begin{aligned}
e^{i t p} & \left.\sum_{\iota=1}^{J} \sum_{j=-\varrho_{\iota}}^{-1} \sum_{k=0}^{\varrho_{\iota}+j} \frac{1}{(-j-1) !}\left(\frac{\partial}{\partial z}+i t\right)^{-j-1} \mathcal{F}_{t \mapsto z}\left\langle v_{k}^{(\iota)}, u\right\rangle\right|_{z=p} f_{\ell_{\iota}+j-k}^{(\iota)} \\
= & e^{i t p} \sum_{\iota=1}^{J} \sum_{j=0}^{\varrho_{\iota}-1}\left(\sum_{k=1}^{\varrho_{\iota}-j} \int_{\mathbb{R}} e^{-i p t^{\prime}} \frac{\left(i t-i t^{\prime}\right)^{k-1}}{(k-1) !}\left\langle v_{\ell_{\iota}-j-k}^{(\iota)}, u\left(t^{\prime}\right)\right\rangle d t^{\prime}\right) f_{j}^{(\iota)},
\end{aligned}
$$

showing that the equation $\operatorname{res}_{p} e^{i t z} a(z) \mathcal{F} u(z)=0$ just amounts to a system of

$$
\sum_{\iota=1}^{J} \varrho_{\iota}=\mathfrak{p}(a(p))
$$

linearly independent moment conditions on the function $u$. These moments are induced by the system

$$
\left(\sum_{k=1}^{\varrho_{\iota}-j} e^{-i p t^{\prime}} \frac{\left(-i t^{\prime}\right)^{k-1}}{(k-1) !} v_{\varrho_{\iota}-j-k}^{(l)}(x)\right)_{\substack{l=1, \ldots, J \\ j=0,1, \ldots, \ell_{\ell}-1}},
$$

each function being in $H^{\infty,-w}(\mathcal{C})$. Hence the lemma follows.

Unless otherwise stated we assume that $a(z) \in \mathcal{M}^{m}(\Xi)$ is parameterdependent elliptic. For $u \in \operatorname{Dom} A$, we define $A u$ by formula (5.1) with any one $\gamma$ in the interval $\left[-w_{-}, w_{+}\right]$, such that the line $\Gamma_{\gamma}$ is free from the poles of $a(z)$.

Proposition 6.6 As defined above, Au is independent of the particular choice of $\gamma$. If moreover a $(z)$ has no pole on the lines $\Gamma_{-w_{-}}$and $\Gamma_{w_{+}}$, then $A u \in H^{s-m, w}(\mathcal{C})$ and the corresponding operator $A: \operatorname{Dom} A \rightarrow H^{s-m, w}(\mathcal{C})$ is continuous.

Proof. The first assertion follows immediately from Lemma 6.4. To prove the second part, we apply Lemma 6.4 once again to obtain

$$
\begin{aligned}
A u(t) & =\frac{1}{2 \pi} \int_{\Gamma_{-w_{-}}} e^{i t z} a(z) \mathcal{F} u(z) d z \\
& =\frac{1}{2 \pi} \int_{\Gamma_{w_{+}}} e^{i t z} a(z) \mathcal{F} u(z) d z
\end{aligned}
$$

for all $t \in \mathbb{R}$. From the first equality it follows, by Proposition 5.2, that $A u \in H^{s-m,-w}-(\mathcal{C})$. On the other hand, the second equality implies that $A u \in H^{s-m, w+}(\mathcal{C})$. We can now invoke Lemma 6.2 to see that $A u$ belongs actually to $H^{s-m, w}(\mathcal{C})$ and

$$
\begin{aligned}
\|A u\|_{H^{s-m, w}(\mathcal{C})} & \leq c\left(\|A u\|_{H^{s-m,-w}-(\mathcal{C})}+\|A u\|_{H^{s-m, w}+(\mathcal{C})}\right) \\
& \leq c\left(\|u\|_{H^{s,-w}-(\mathcal{C})}+\|u\|_{H^{s, w}+(\mathcal{C})}\right) \\
& \leq c\|u\|_{H^{s, w}(\mathcal{C})}
\end{aligned}
$$


the constant $c$ being independent of $u$ while not necessarily the same in different applications. This completes the proof.

We are now in a position to study the inhomogeneous equation $A u=f$ for an unknown function $u \in \operatorname{Dom} A$, where $f \in H^{s-m, w}(\mathcal{C})$. As follows, this equation is solvable for $f$ in a closed subspace of finite codimension in $H^{s-m, w}(\mathcal{C})$.

Theorem 6.7 Let a $(z)$ have no singular value on the lines $\Gamma_{-w_{-}}$and $\Gamma_{w_{+}}$. Suppose $f \in H^{s-m, w}(\mathcal{C})$, where $s \geq \max (0, m)$. Then, in order that there exist a function $u \in \operatorname{Dom} A$ satisfying $A u=f$ it is necessary and sufficient that

$$
\operatorname{res}_{p} e^{i t z} a^{-1}(z) \mathcal{F} f(z)=0 \quad \text { for } \quad-w_{-}<\Im p<w_{+} .
$$

Proof. Necessity. If $f=A u$ for some function $u \in H^{s, w}(\mathcal{C})$, then $\mathcal{F} f(z)=a(z) \mathcal{F} u(z)$, which is clear from Lemma 5.1. As $\mathcal{F} u(z)$ is holomorphic in the strip $-w_{-}<\Im z<w_{+}$, we get

$$
\begin{aligned}
\operatorname{res}_{p} e^{i t z} a^{-1}(z) \mathcal{F} f(z) & =\operatorname{res}_{p} e^{i t z} \mathcal{F} u(z) \\
& =0
\end{aligned}
$$

for each pole $p$ of $a^{-1}(z)$ with $-w_{-}<\Im p<w_{+}$. This is precisely our assertion.

Sufficiency. Conversely, let $f \in H^{s-m, w}(\mathcal{C}), s \geq \max (0, m)$, satisfy condition (6.5). By Lemma 6.4 we can assert that the integral

$$
u(t)=\int_{\Gamma_{\gamma}} e^{i t z} a^{-1}(z) \mathcal{F} f(z) d z, \quad t \in \mathbb{R},
$$

is independent of the particular choice of $\gamma$ in the interval $\left[-w_{-}, w_{+}\right]$, provided that the line $\Gamma_{\gamma}$ does not meet any pole of $a^{-1}(z)$. Taking $\gamma$ to be $-w_{-}$and $w_{+}$, we conclude from Proposition 5.2 that $u$ belongs both to $H^{s,-w}-(\mathcal{C})$ and $H^{s, w}+(\mathcal{C})$. Lemma 6.2 now shows that $u \in H^{s, w}(\mathcal{C})$. As $\mathcal{F} u(z)=a^{-1}(z) \mathcal{F} f(z)$ and $\mathcal{F} f(z)$ is holomorphic for $-w_{-}<\Im z<w_{+}$, it is immediate that $u \in \operatorname{Dom} A$. Moreover, applying Proposition 5.3 gives $A u=f$, and the proof is complete.

Note that we have actually proved that the equation $A u=f$ has at most one solution in Dom $A$. In fact, under moment conditions (6.5), the solution is given by formula (5.3) with any one $\gamma \in\left[-w_{-}, w_{+}\right]$.

Corollary 6.8 Assume that $a(z)$ is invertible on the lines $\Gamma_{-w_{-}}$and $\Gamma_{w_{+}}$. Then, the operator $A: \operatorname{Dom} A \rightarrow H^{s-m, w}(\mathcal{C})$ is injective and has a closed range of finite codimension,

$$
\operatorname{codim} \operatorname{Ran} A=\sum_{-w_{-}<\Im p<w_{+}} \mathfrak{n}(a(p))
$$


Proof. Indeed, combining Theorem 6.7 and Lemma 6.5 we conclude that

$$
\begin{aligned}
\operatorname{codim} \operatorname{Ran} A & =\operatorname{codim} \operatorname{Dom} A^{-1} \\
& =\sum_{-w_{-}<\Im p<w_{+}} \mathfrak{p}\left(a^{-1}(p)\right) \\
& =\sum_{-w_{-}<\Im p<w_{+}} \mathfrak{n}(a(p)),
\end{aligned}
$$

the last equality being a consequence of what has been proved in Section 2 . This establishes the formula.

The corollary says that the operator $A: \operatorname{Dom} A \rightarrow H^{s-m, w}(\mathcal{C})$ is Fredholm, and yields information about the index of $A$.

\section{Transposed equation}

We keep the notation of the previous section. In particular, $a(z) \in \mathcal{M}^{m}(\Xi)$ is assumed to be parameter-dependent elliptic.

Our goal is to assign a natural pseudodifferential operator $A$ to $a(z)$ which acts in Sobolev spaces $H^{s, w}(\mathcal{C})$ with weight data $w=\left(w_{-}, w_{+}\right)$satisfying $a<w_{+}<-w_{-}<b$. Note that if $H^{s, w}(\mathcal{C}) \hookrightarrow H^{s, \gamma}(\mathcal{C})$ for some $\gamma \in \mathbb{R}$, then $-\gamma \leq w_{-}$and $\gamma \leq w_{+}$implying $-w_{-} \leq w_{+}$. Consequently, in the case $-w_{-}>w_{+}$we can no longer define $A$ by formula (5.1). Moreover, the Fourier transform of a function $u \in H^{s, w}$ is no longer holomorphic in any strip, which results in the rigidity of the contour of integration. Of course, no problem arises in case $a(z)$ is a polynomial function of $z$, i.e., for differential operators on the cylinder $\mathcal{C}$.

To cope with these difficulties, we invoke familiar duality arguments. For this purpose, let us have look at the transpose of the operator $A$ given by (5.1).

Proposition 7.1 Suppose that $a(z)$ has no pole on a line $\Gamma_{\gamma}$, where $\gamma \in(a, b)$. For any $s \in \mathbb{R}$, the transpose $A^{\prime}: H^{-s+m,-\gamma}(\mathcal{C}) \rightarrow H^{-s,-\gamma}(\mathcal{C})$ of the mapping of Proposition 5.2 is induced by

$$
A^{\prime} g(t)=\frac{1}{2 \pi} \int_{\Gamma_{-\gamma}} e^{i t z} a^{\prime}(-z) \mathcal{F} g(z) d z, \quad t \in \mathbb{R} .
$$

Proof. It suffices to specify $A^{\prime} g$ for $g \in C_{\text {comp }}^{\infty}(\mathcal{C})$. If $u \in C_{\text {comp }}^{\infty}(\mathcal{C})$, then

$$
\begin{aligned}
\langle g, A u\rangle & =\int_{\mathbb{R}}\left\langle g(t), \frac{1}{2 \pi} \int_{\Gamma_{\gamma}} e^{i t z} a(z) \mathcal{F} u(z) d z\right\rangle d t \\
& =\int_{\mathbb{R}}\left\langle\frac{1}{2 \pi} \int_{\Gamma_{\gamma}} e^{-i t^{\prime} z} a^{\prime}(z) \mathcal{F} g(-z) d z, u\left(t^{\prime}\right)\right\rangle d t^{\prime}
\end{aligned}
$$


as is easy to check. Changing the variable in the inner integral by $z \mapsto-z$, we arrive at (7.1), as desired.

Thus, the transpose of (5.1) is actually given by the same formula, with $a(z)$ and $\gamma$ replaced by $a^{\prime}(-z)$ and $-\gamma$, respectively. If $-w_{-}>w_{+}$, then the weight data $-w=\left(-w_{-},-w_{+}\right)$meets the condition of Lemma 6.2. Hence we may apply the techniques of the previous section to study the operator $A^{\prime}: H^{-s+m,-w}(\mathcal{C}) \rightarrow H^{-s,-w}(\mathcal{C})$ related to the symbol function $a^{\prime}(-z) \in \mathcal{M}^{m}(-\Xi)$ via $(7.1)$. Recall that $a^{\prime}(-z)$ is parameter-dependent elliptic, for so is $a(z)$. It follows that, for $s \leq \min (0, m)$, the domain of $A^{\prime}$, i.e.,

$\operatorname{Dom} A^{\prime}=\left\{g \in H^{-s+m,-w}(\mathcal{C}): \operatorname{res}_{p} e^{i t z} a^{\prime}(-z) \mathcal{F} g(z)=0\right.$ for $\left.w_{-}<\Im p<-w_{+}\right\}$

is a closed subspace of finite codimension in $H^{-s+m,-w}(\mathcal{C})$. More precisely, we have

$$
\begin{aligned}
\operatorname{codim} \operatorname{Dom} A^{\prime} & =\sum_{w_{-}<\Im p<-w_{+}} \mathfrak{p}\left(a^{\prime}(-p)\right) \\
& =\sum_{w_{+}<\Im p<-w_{-}} \mathfrak{p}(a(p)),
\end{aligned}
$$

the last equality being a consequence of Corollary 3.3. By Corollary 6.8, if $a(z)$ is invertible on both the lines $\Gamma_{-w_{-}}$and $\Gamma_{w_{+}}$, then the operator $A^{\prime}:$ Dom $A^{\prime} \rightarrow H^{-s,-w}(\mathcal{C})$ is injective and has a closed range of finite codimension

$$
\begin{aligned}
\operatorname{codim} \operatorname{Ran} A^{\prime} & =\sum_{w_{-}<\Im p<-w_{+}} \mathfrak{n}\left(a^{\prime}(-p)\right) \\
& =\sum_{w_{+}<\Im p<-w_{-}} \mathfrak{n}(a(p)),
\end{aligned}
$$

the second equality being due to Corollary 3.3. Were Dom $A^{\prime}$ equal to $H^{-s+m,-w}(\mathcal{C})$, we would define $A: H^{s, w}(\mathcal{C}) \rightarrow H^{s-m, w}(\mathcal{C})$ to be the transpose of the mapping $A^{\prime}: H^{-s+m,-w}(\mathcal{C}) \rightarrow H^{-s,-w}(\mathcal{C})$, thus arriving at a surjective operator whose null-space is the annihilator of $\operatorname{Ran} A^{\prime}$ in $H^{s, w}(\mathcal{C})$. Such is the case if $a(z)$ has no pole in the strip $w_{+}<\Im_{z}<-w_{-}$, in particular, for differential operators on the cylinder. However, in the general case we have to take more care on the definition of $A$ as the dual of Dom $A^{\prime}$ can not be identified within $H^{s-m, w}(\mathcal{C})$.

Lemma 7.2 Let $H_{1}, H_{2}$ be reflexive Fréchet spaces. Suppose that $A^{\prime}:$ Dom $A^{\prime} \rightarrow H_{1}^{\prime}$ is a continuous mapping whose domain is a complemented subspace of $H_{2}^{\prime}$. Then, for each $u \in H_{1}$ there is unique element $f \in H_{2}$ such that

$$
\begin{array}{lll}
\langle g, f\rangle=\left\langle A^{\prime} g, u\right\rangle & \text { for all } & g \in \operatorname{Dom} A^{\prime} ; \\
\langle g, f\rangle=0 & \text { for all } & g \in H_{2}^{\prime} \ominus \operatorname{Dom} A^{\prime} .
\end{array}
$$


Here, we use the customary notation $H^{\prime}$ for the dual of a topological vector space $H$.

Proof. Indeed, pick a topological complement $H_{2}^{\prime} \ominus$ Dom $A^{\prime}$ of the subspace Dom $A^{\prime}$ in $H_{2}^{\prime}$. Denote by $\pi$ the projection of $H_{2}^{\prime}$ onto Dom $A^{\prime}$ parallel to $H_{2}^{\prime} \ominus \operatorname{Dom} A^{\prime}$. Given $u \in H_{1}$, define a functional $f$ on $H_{2}^{\prime}$ by

$$
\langle g, f\rangle=\left\langle A^{\prime} \pi g, u\right\rangle
$$

for $g \in H_{2}^{\prime}$. As both $A^{\prime}$ and $\pi$ are continuous, we conclude that $f$ is a continuous linear functional on $H_{2}^{\prime}$, and so $f$ can be identified with an element of $H_{2}$. Obviously, $f$ satisfies (7.4). On the other hand, if $f_{1}, f_{2} \in H_{2}$ satisfy (7.4), then

$$
\begin{aligned}
\left\langle g, f_{1}-f_{2}\right\rangle & =\left\langle\pi g, f_{1}-f_{2}\right\rangle+\left\langle(1-\pi) g, f_{1}-f_{2}\right\rangle \\
& =0
\end{aligned}
$$

for all $g \in H_{2}^{\prime}$, whence $f_{1}=f_{2}$. The proof is complete.

Setting $A u=f$, we thus get a linear operator $H_{1} \rightarrow H_{2}$. A simple argument of functional analysis shows that $A$ is continuous. However, it is worth pointing out that the definition of $A$ depends on the particular choice of the topological complement of Dom $A^{\prime}$ in $H_{2}^{\prime}$, unless Dom $A^{\prime}=H_{2}^{\prime}$.

Applying this abstract scheme to our problem, we arrive at a continuous linear operator $A: H^{s, w}(\mathcal{C}) \rightarrow H^{s-m, w}(\mathcal{C})$, for $s \leq \min (0, m)$. By definition, we have

$$
\begin{array}{ll}
\langle g, A u\rangle=\left\langle A^{\prime} g, u\right\rangle & \text { for } \quad g \in \operatorname{Dom} A^{\prime} ; \\
\langle g, A u\rangle=0 & \text { for } g \in H^{-s+m,-w}(\mathcal{C}) \ominus \operatorname{Dom} A^{\prime},
\end{array}
$$

provided $u \in H^{s, w}(\mathcal{C})$. If $a(z)$ is a polynomial function of $z$ with values in $\Psi_{\mathrm{cl}}^{m}(X)$, then $A$ agrees with the usual extension of a differential operator to spaces of distributions.

Theorem 7.3 Let $a(z)$ be invertible on the lines $\Gamma_{-w_{-}}$and $\Gamma_{w_{+}}$. As defined by $(7.5), A: H^{s, w}(\mathcal{C}) \rightarrow H^{s-m, w}(\mathcal{C})$ is a Fredholm operator. Moreover, the null-space of $A$ is of dimension codim Ran $A^{\prime}$ and the range of $A$ is of codimension codim Dom $A^{\prime}$.

Proof. By $(7.5), u \in H^{s, w}(\mathcal{C})$ satisfies $A u=0$ if and only if $u$ belongs to the annihilator of $\operatorname{Ran} A^{\prime}$ in $H^{s, w}(\mathcal{C})$. Hence it follows that the dimension of the null-space of $A$ is equal to the codimension of Ran $A^{\prime}$, which is finite. On the other hand, since $A^{\prime}: \operatorname{Dom} A^{\prime} \rightarrow H^{-s,-w}(\mathcal{C})$ is injective, we easily deduce from (7.5) that in order that the equation $A u=f$ be solvable it is necessary and sufficient that $f$ belong to the annihilator of $H^{-s+m,-w}(\mathcal{C}) \ominus \operatorname{Dom} A^{\prime}$ in $H^{s-m, w}(\mathcal{C})$. Therefore, the codimension of the range of $A$ coincides with the codimension of Dom $A^{\prime}$, which proves the theorem.

We finish this section with a description of solutions of the homogeneous equation $A u=0$. 
Corollary 7.4 To each characteristic value $p$ of the symbol a $(z)$ in the strip $w_{+}<\Im z<-w_{-}$there correspond $\mathfrak{n}(a(p))$ linearly independent solutions of $A u=0$, namely

$$
\left(\sum_{k=1}^{r_{i}-j} e^{i p t} \frac{(i t)^{k-1}}{(k-1) !} u_{r_{i}-j-k}^{(i)}(x)\right)_{\substack{i=1, \ldots, I \\ j=0,1, \ldots, r_{i}-1}}
$$

where $\left(u_{j}^{(i)}\right)$ is a canonical system of eigenfunctions and associated functions of $a(z)$ at $p$, as guaranteed by Proposition 4.2.

It is a simple matter to verify that each function of the form (7.6) belongs to $H^{\infty, w}(\mathcal{C})$, provided that $w_{+}<\Im p<-w_{-}$(cf. (6.4)).

Proof. To highlight the role of canonical systems of eigenfunctions and associated functions, we give the proof only for polynomial functions $a(z)$. The general case needs handling with greater care for we define $A$ by a dual argument (cf. (7.5)). For the proof, fix $i=1, \ldots, I$. By definition, there is a root function $u^{(i)}(z)$ of multiplicity $r_{i}$ for $a(z)$ at the point $p$, such that

$$
\frac{1}{j !}\left(\frac{\partial}{\partial z}\right)^{j} u^{(i)}(p)=u_{j}^{(i)}(x)
$$

for $j=0,1, \ldots, r_{i}-1$. Hence it follows that

$$
\begin{aligned}
\sum_{k=1}^{r_{i}-j} e^{i p t} \frac{(i t)^{k-1}}{(k-1) !} u_{r_{i}-j-k}^{(i)}(x) & =\left.\sum_{k=1}^{r_{i}-j} \frac{1}{(k-1) !}\left(\frac{\partial}{\partial z}\right)^{k-1} e^{i z t}\right|_{z=p} u_{r_{i}-j-k}^{(i)}(x) \\
& =\left.\frac{1}{\left(r_{i}-j-1\right) !}\left(\frac{\partial}{\partial z}\right)^{r_{i}-j-1}\left(e^{i z t} u^{(i)}(z)\right)\right|_{z=p}
\end{aligned}
$$

the last equality being a consequence of the Leibniz formula. When applying the operator $A$ to the right-hand side of this equality, we may interchange $A$ and the derivative in $z$. This implies

$$
\begin{aligned}
A\left(\sum_{k=1}^{r_{i}-j} e^{i p t} \frac{(i t)^{k-1}}{(k-1) !} u_{r_{i}-j-k}^{(i)}(x)\right) & =\left.\frac{1}{\left(r_{i}-j-1\right) !}\left(\frac{\partial}{\partial z}\right)^{r_{i}-j-1} A\left(e^{i z t} u^{(i)}(z)\right)\right|_{z=p} \\
& =\left.\frac{1}{\left(r_{i}-j-1\right) !}\left(\frac{\partial}{\partial z}\right)^{r_{i}-j-1}\left(e^{i z t} a(z) u^{(i)}(z)\right)\right|_{z=p}
\end{aligned}
$$

the right-hand side being zero for each $j=0,1, \ldots, r_{i}-1$ because $a(z) u^{(i)}(z)$ vanishes up to order $r_{i}-1$ at the point $p$. To complete the proof it suffices to note, by Theorem 7.3, that system (7.6) encompasses the whole contribution of $p$ to the null-space of $A$. 


\section{Index}

Let $a(z) \in \mathcal{M}^{m}(\Xi)$ be a parameter-dependent elliptic function with values in $\Psi_{\mathrm{cl}}^{m}(X)$. Given any weight data $w=\left(w_{-}, w_{+}\right)$with $w_{-}, w_{+} \in(a, b)$, we assign a pseudodifferential operator $A: H^{s, w}(\mathcal{C}) \rightarrow H^{s-m, w}(\mathcal{C})$ to $a(z)$, as described above. Recall that in the case $-w_{-}<w_{+}$the operator $A$ is not defined on all of $H^{s, w}(\mathcal{C})$ unless the symbol $a(z)$ has no pole in the strip $-w_{-}<\Im z<w_{+}$. To arrive at an operator defined on the entire space $H^{s, w}(\mathcal{C})$ we fix a topological complement of the domain of $A$ in $H^{s, w}(\mathcal{C})$ and compose $A$ with the projection of $H^{s, w}(\mathcal{C})$ onto Dom $A$. By abuse of notation, we continue to write $A$ for the resulting operator whose null-space is $H^{s, w}(\mathcal{C}) \ominus \operatorname{Dom} A$. Corollary 6.8 and Theorem 7.3 state that if $a(z)$ has no singular values on the lines $\Gamma_{-w_{-}}$and $\Gamma_{w_{+}}$, then $A$ is a Fredholm operator. Hence the index of $A$ is well-defined and independent of the choice of $s$ provided $s$ meets the condition above. The following theorem provides us with an explicit formula for the index.

Theorem 8.1 Suppose that $a(z)$ is invertible on the lines $\Gamma_{-w_{-}}$and $\Gamma_{w_{+}}$. Then,

$$
\text { ind } A=\operatorname{tr}\left(\frac{1}{2 \pi i} \int_{\Gamma_{w_{+}}} a^{-1}(z) a^{\prime}(z) d z-\frac{1}{2 \pi i} \int_{\Gamma_{-w_{-}}} a^{-1}(z) a^{\prime}(z) d z\right) .
$$

We emphasise that the integrals on the right-hand of (8.1) are divergent while their sum makes sense to be explained in the proof. Moreover, the operators $a^{-1}(z) a^{\prime}(z)$ are not of trace class on $X$ unless $X$ is zero-dimensional. However, the operator-valued function $a^{-1}(z) a^{\prime}(z)$ is holomorphic everywhere in the strip between $\Gamma_{-w_{-}}$and $\Gamma_{w_{+}}$, except possibly at a finite number of points which are either poles or characteristic values of $a(z)$. Thus, only the principal parts of Laurent expansions of this function near singular values contribute to the sum of the integrals, as is clear from the residue formula. We then invoke the fact that these principal parts take their values in the space of smoothing operators on $X$.

Proof. Indeed, from what has been proved in Sections 6 and 7 it follows that

$$
\text { ind } \begin{aligned}
A & =\sum_{-w_{-}<\Im p<w_{+}} \mathfrak{p}(a(p))-\sum_{-w_{-}<\Im p<w_{+}} \mathfrak{n}(a(p)) \\
& =-\sum_{-w_{-}<\Im p w_{+}} \mathfrak{m}(a(p)),
\end{aligned}
$$

if $-w_{-} \leq w_{+}$, and

$$
\text { ind } \begin{aligned}
A & =\sum_{w_{+}<\Im p<-w_{-}} \mathfrak{n}(a(p))-\sum_{w_{+}<\Im p<-w_{-}} \mathfrak{p}(a(p)) \\
& =\sum_{w_{+}<\Im p<-w_{-}} \mathfrak{m}(a(p)),
\end{aligned}
$$


if $-w_{-}>w_{+}$. We now compute the sum on the right-hand sides in another way. To this end, for $T>0$, denote by $Q_{T}$ the rectangle with vertices $-T-i w_{-}, T-i w_{-}, T+i w_{+}$and $-T+i w_{+}$(cf. Fig. 1$)$. Let $T \gg 1$ be so chosen that $Q_{T}$ contains all singular values of $a(z)$ in the strip between $\Gamma_{-w_{-}}$and $\Gamma_{w_{+}}$. Combining Corollary 3.2 with the residue formula we can assert that

$$
\frac{1}{2 \pi i} \operatorname{tr} \int_{\partial Q_{T}} a^{-1}(z) a^{\prime}(z) d z=\sum_{w_{+}<\Im p<-w_{-}} \mathfrak{m}(a(p))
$$

for $T$ large enough. Thus, the integral on the left is equal to the index of $A$, up to the sign of $-w_{-}-w_{+}$. Letting $T \rightarrow \infty$ we arrive at (8.1), and the proof is complete.

Formula 8.1 is a rather particular case of the theorem on the logarithmic residue for meromorphic operator-valued functions due to Gokhberg and Sigal [GS71]. Our viewpoint sheds some new light on the general index theorem for elliptic pseudodifferential operators on manifolds with conical points given in [FST97]. Note that explicit formulas (7.6) for solutions of $A u=0$ along with those for solutions of $A^{\prime} g=0$ can be of use to derive a simple Lefschetz fixed point formula for the operator $A$, generalising the index formula (8.1) (cf. Theorem 4.1 in [GS71]). 


\section{References}

[AN63] S. Agmon and L. Nirenberg, Properties of solutions of ordinary differential equations in Banach space, Comm. Pure Appl. Math. 16 (1963), no. 2, 121-239.

[AV64] M. S. Agranovich and M. I. Vishik, Elliptic problems with parameter and parabolic problems of general type, Uspekhi Mat. Nauk 19 (1964), no. 3, 53-160.

[Ble69] P. M. Blekher, Operators depending meromorphically on a parameter, Vestnik Mosk. Univ., Mat., Meh. 24 (1969), no. 5, 30-36.

[Eni69] V. M. Eni, On the multiplicity of a characteristic value of an operator bundle, Mat. Issled. 4 (1969), no. 2, 32-41.

[Evg61] M. A. Evgrafov, Structure of solutions of exponential growth for some operator equations, Trudy Mat. Inst. im. V.A. Steklova 60 (1961), 145-180.

[FST97] B. V. Fedosov, B.-W. Schulze, and N. N. Tarkhanov, The Index of Elliptic Operators on Manifolds with Conical Points, Preprint 97/24, Univ. Potsdam, Potsdam, August 1997.

[Gok51] I. Ts. Gokhberg, On linear operators depending analytically on a parameter, Dokl. Akad. Nauk SSSR 78 (1951), 629-632.

[GS71] I. Ts. Gokhberg and E. I. Sigal, An operator generalisation of the logarithmic residue theorem and the theorem of Rouché, Mat. Sb. 84 (126) (1971), no. 4, 607-629 (Russian). English transl. Math. USSR Sbornik 13 (1971), No. 4.

[Kel51] M. V. Keldysh, On the characteristic values and characteristic functions of certain classes of non-selfadjoint equations, Dokl. Akad. Nauk SSSR 77 (1951), 11-14.

[Kon67] V. A. Kondrat'ev, Boundary value problems for elliptic equations in domains with conical points, Trudy Mosk. Mat. Obshch. 16 (1967), 209-292.

[KT69] S. G. Krein and V. P. Trofimov, Holomorphic operator-valued functions of several complex variables, Funkts. Analiz 3 (1969), no. $4,85-86$.

[MKR97] V. G. Maz'ya, V. Kozlov, and J. Rossmann, Point Boundary Singularities in Elliptic Theory, AMS, Providence, R.I., 1997. 
[MM83] R. B. Melrose and G. A. Mendoza, Elliptic operators of totally characteristic type. Preprint MSRI 047-83, MSRI, Berkeley, 1983.

[MP72] V. G. Maz'ya and B. A. Plamenevskii, On the asymptotic behavior of solutions of differential equations in Hilbert space, Math. USSR Izvestija 6 (1972), no. 5, 1067-1116.

[MS70] A. S. Markus and E. I. Sigal, On the multiplicity of a characteristic value of an analytic operator-valued function, Mat. Issled. 5 (1970), no. 3, 129-147.

[Pla89] B. A. Plamenevskii, Algebras of Pseudodifferential Operators, Kluwer Academic Publishers, Dordrecht NL, 1989.

[Sch91] B.-W. Schulze, Pseudo-Differential Operators on Manifolds with Singularities, North-Holland, Amsterdam, 1991.

[Sch98] B.-W. Schulze, Boundary Value Problems and Singular Pseudo-Differential Operators, J. Wiley, Chichester, 1998.

[Shu87] M. A. Shubin, Pseudodifferential Operators and Spectral Theory, Springer-Verlag, Berlin et al., 1987.

[Sig70] E. I. Sigal, On the multiplicity of a characteristic value of the product of operator-valued functions, Mat. Issled. 5 (1970), no. 1, 118-127.

[SS94] E. Schrohe and B.-W. Schulze, Boundary value problems in Boutet de Monvel's algebra for manifolds with conical singularities. I, Pseudodifferential Operators and Mathematical Physics. Advances in Partial Differential Equations 1, Akademie-Verlag, Berlin, 1994, pp. 97-209.

[SS95] E. Schrohe and B.-W. Schulze, Boundary value problems in Boutet de Monvel's algebra for manifolds with conical singularities. II, Boundary Value Problems, Deformation Quantization, Schrödinger Operators. Advances in Partial Differential Equations 2, Akademie-Verlag, Berlin, 1995, pp. 70-205.

[Ste70] B. Yu. Sternin, Quasi-elliptic equations in an infinite cylinder, Dokl. Akad. Nauk SSSR 194 (1970), 1025-1028. 